\title{
Synthesis of Site-Specifically Labeled Arachidonic Acids as Mechanistic Probes for Prostaglandin H Synthase
}

\author{
Sheng Peng, Chris M. McGinley, and Wilfred A. van der Donk* \\ Roger Adams Laboratory, Department of Chemistry, University of Illinois, 600 S. Mathews Avenue, \\ Urbana, Illinois, 61801
}

Supporting Information

$\left[10,10-{ }^{2} \mathrm{H}_{2}\right]$-Arachidonic Acid

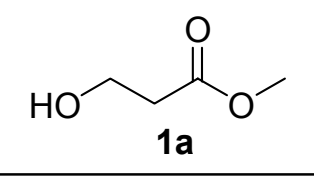

3-Hydroxy-propionic acid methyl ester (1a)

A dry flask was charged with $\beta$-propiolactone $(1.00 \mathrm{~g}, 13.88 \mathrm{mmol})$ and $25 \mathrm{~mL}$ of methanol was added. The reaction mixture was stirred at room temperature for $72 \mathrm{~h}$. The solvent was removed and the resulting oil purified by flash chromatography $(10 \%$ IPA/Hex, $R_{\mathrm{f}}=0.29$ in $\left.20 \% \mathrm{IPA} / \mathrm{Hex}\right)$ yielding $910 \mathrm{mg}(62 \%)$ of $1 \mathrm{a}$ as a colorless oil. ${ }^{1} \mathrm{H}$ $\operatorname{NMR}\left(500 \mathrm{MHz}, \mathrm{CDCl}_{3}\right) \delta 2.60(\mathrm{t}, \mathrm{J}=5.6 \mathrm{~Hz}, 2 \mathrm{H}), 3.74(\mathrm{~s}, 3 \mathrm{H}), 3.88(\mathrm{q}, \mathrm{J}=5.7 \mathrm{~Hz}, 2 \mathrm{H})$. Spectral data matched that reported in the literature. ${ }^{1}$

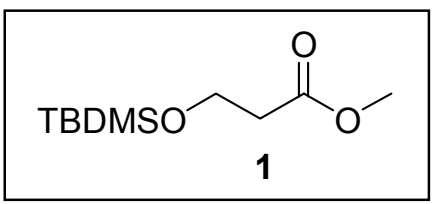

3-(tert-Butyl-dimethyl-silanyloxy)-propionic acid methyl ester (1)

A dry flask was charged with imidazole $(1.71 \mathrm{~g}, 24.4 \mathrm{mmol})$ and tert-butyldimethylsilyl chloride $(3.71 \mathrm{~g}, 24.4 \mathrm{mmol})$. After purging with argon, $40 \mathrm{~mL}$ of $\mathrm{CH}_{2} \mathrm{Cl}_{2}$ was added. This suspension was slowly added to a solution of 3-hydroxy-propionic acid methyl ester (910 mg, $8.71 \mathrm{mmol}$ ) in $10 \mathrm{~mL}$ of $\mathrm{CH}_{2} \mathrm{Cl}_{2}$ at $0{ }^{\circ} \mathrm{C}$. The reaction was stirred for $2 \mathrm{~h}$, then slowly warmed to room temperature overnight. The reaction was poured into $50 \mathrm{~mL}$ of water, and extracted with $\mathrm{CH}_{2} \mathrm{Cl}_{2}(3 \times 50 \mathrm{~mL})$. The combined organic layers were dried with $\mathrm{MgSO}_{4}$, filtered and concentrated. The oil was purified by flash chromatography ( $5 \%$ EtOAc/ Hex, $\mathrm{R}_{\mathrm{f}}=0.44$ in $10 \%$ EtOAc in Hex) yielding $1.94 \mathrm{~g}(100 \%)$ of $\mathbf{1}$ as a clear oil. ${ }^{1} \mathrm{H}$ NMR $\left(500 \mathrm{MHz}, \mathrm{CDCl}_{3}\right) \delta 0.07(\mathrm{~s}, 6 \mathrm{H}), 0.89(\mathrm{~s}, 9 \mathrm{H}), 2.55(\mathrm{t}, \mathrm{J}=6.4 \mathrm{~Hz}, 2 \mathrm{H})$, $3.70(\mathrm{~s}, 3 \mathrm{H}), 3.91(\mathrm{t}, \mathrm{J}=6.4 \mathrm{~Hz}, 2 \mathrm{H}) .{ }^{13} \mathrm{C} \mathrm{NMR}\left(125.6 \mathrm{MHz}, \mathrm{CDCl}_{3}\right) \delta-5.3\left(\mathrm{CH}_{3}\right), 18.4$ $(\mathrm{Cq}), 26.0\left(\mathrm{CH}_{3}\right), 38.1\left(\mathrm{CH}_{2}\right), 51.7\left(\mathrm{CH}_{3}\right), 59.3\left(\mathrm{CH}_{2}\right), 172.5(\mathrm{Cq})$. Spectral data matched that reported in the literature. ${ }^{1}$ 


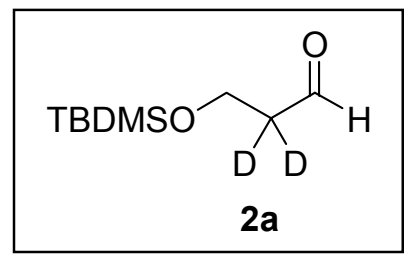

\section{[2- $\left.{ }^{2} \mathbf{H}_{2}\right]$-3-(tert-Butyl-dimethyl-silanyloxy)-propionaldehyde (2a)}

A dry flask was charged with Na metal $(90.2 \mathrm{mg}, 3.92 \mathrm{mmol})$. After purging with Ar, 20 $\mathrm{mL}$ of $\mathrm{MeOD}$ was added and the mixture stirred until the metal dissolved. This solution was cannulated into a degassed flask containing $1(808 \mathrm{mg}, 3.70 \mathrm{mmol})$. The solution was stirred at room temperature for $6 \mathrm{~h}$. The reaction was neutralized with $\mathrm{DCl}$ and poured into $75 \mathrm{~mL}$ of water and extracted with diethyl ether $(3 \times 50 \mathrm{~mL})$. The combined organic layers were dried with $\mathrm{MgSO}_{4}$, filtered and concentrated. The resulting oil was purified by flash chromatography $\left(5 \%\right.$ EtOAc/ Hex, $\mathrm{R}_{\mathrm{f}}=0.44$ in $10 \%$ EtOAc in Hex $)$, yielding $666.1 \mathrm{mg}(82 \%)$ of $\left[2-{ }^{2} \mathrm{H}_{2}\right]-3$-(tert-butyl-dimethyl-silanyloxy)-propionic acid methyl ester as a clear oil. ${ }^{1} \mathrm{H}$ NMR $\left(500 \mathrm{MHz}, \mathrm{CDCl}_{3}\right) \delta 0.06(\mathrm{~s}, 6 \mathrm{H}), 0.88(\mathrm{~s}, 9 \mathrm{H}), 3.69$ $(\mathrm{s}, 3 \mathrm{H}), 3.90(\mathrm{~s}, 2 \mathrm{H}) .{ }^{13} \mathrm{C} \mathrm{NMR}\left(125.6 \mathrm{MHz}, \mathrm{CDCl}_{3}\right) \delta-5.28\left(\mathrm{CH}_{3}\right), 22.84(\mathrm{Cq}), 26.0$ $\left(\mathrm{CH}_{3}\right), 51.6\left(\mathrm{CH}_{3}\right), 59.2\left(\mathrm{CH}_{2}\right), 172.5(\mathrm{Cq})$. IR: 2956, 1746, 1473, 1257. HRMS (CI, $\mathrm{M}+1$ ) for $\mathrm{C}_{10} \mathrm{H}_{21} \mathrm{D}_{2} \mathrm{O}_{3}$ Si calcd 221.1542, found 221.1542.

A dry flask was charged with the methyl ester $(294.2 \mathrm{mg}, 1.34 \mathrm{mmol})$ and purged with argon. $\mathrm{CH}_{2} \mathrm{Cl}_{2}(15 \mathrm{~mL})$ was added and the solution cooled to $-78{ }^{\circ} \mathrm{C}$. A $1.0 \mathrm{M}$ solution of DIBAL-H $(1.48 \mathrm{~mL}, 1.48 \mathrm{mmol})$ was added dropwise and the solution was stirred for $90 \mathrm{~min}$. The reaction was quenched with $0.5 \mathrm{~mL}$ of methanol and warmed to room temperature. Diethylether $(20 \mathrm{~mL})$ and a saturated solution of sodium potassium tartrate $(10 \mathrm{~mL})$ was added and the two-phase mixture was stirred for $45 \mathrm{~min}$. The solution was extracted with diethyl ether $(3 \times 50 \mathrm{~mL})$, dried with $\mathrm{MgSO}_{4}$, filtered and concentrated. The resulting oil was purified by flash chromatography $\left(25 \%\right.$ ether in pentane, $\mathrm{R}_{\mathrm{f}}=0.63$ in $20 \%$ EtOAc in Hex) yielding $245.1 \mathrm{mg}(96 \%)$ of $\mathbf{2 a}$ as a clear oil. ${ }^{1} \mathrm{H}$ NMR $(500 \mathrm{MHz}$, $\left.\mathrm{CDCl}_{3}\right) \delta 0.06(\mathrm{~s}, 6 \mathrm{H}), 0.88(\mathrm{~s}, 9 \mathrm{H}), 3.98(\mathrm{~s}, 2 \mathrm{H}), 9.80(\mathrm{~s}, 1 \mathrm{H}) .{ }^{13} \mathrm{C} \mathrm{NMR}(125.6 \mathrm{MHz}$, $\left.\mathrm{CDCl}_{3}\right) \delta-5.3\left(\mathrm{CH}_{3}\right), 18.4(\mathrm{Cq}), 25.9\left(\mathrm{CH}_{3}\right), 51.7\left(\mathrm{CH}_{3}\right), 59.2\left(\mathrm{CH}_{2}\right), 172.5(\mathrm{Cq})$. IR: 2958, 2859, 1731, 1472, 1257. HRMS (CI, M+1) for $\mathrm{C}_{9} \mathrm{H}_{19} \mathrm{D}_{2} \mathrm{O}_{2} \mathrm{Si}$ calcd 191.1436, found 191.1438 .

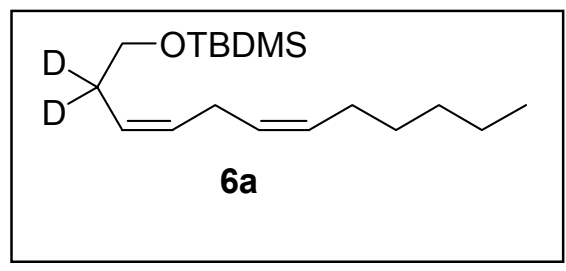

\section{[2- $\left.{ }^{2} \mathbf{H}_{2}\right]$-tert-Butyl-dimethyl-undeca-3,6-dienyloxy-silane (6a)}

A dry flask was charged with phosphonium salt $5(222.6 \mathrm{mg}, 0.47 \mathrm{mmol})$. After purging with argon, $15 \mathrm{~mL}$ of THF was added and the mixture was cooled to $0{ }^{\circ} \mathrm{C}$. A $1.0 \mathrm{M}$ solution of NaHMDS in THF $(450 \mu \mathrm{L}, 0.45 \mathrm{mmol})$ was added and the reaction mixture was stirred for $15 \mathrm{~min}$. The reaction was cooled to $-78{ }^{\circ} \mathrm{C}$, and $2 \mathrm{a}(41.4 \mathrm{mg}, 0.21 \mathrm{mmol})$ in $5 \mathrm{~mL}$ of THF was added dropwise. The reaction was stirred at $-78{ }^{\circ} \mathrm{C}$ for $3 \mathrm{~h}$, then slowly warmed to room temperature overnight. The solution was poured into water and extracted with diethyl ether $(4 \times 50 \mathrm{~mL})$. The combined organic layers were washed with water $(1 \times 60 \mathrm{~mL})$, dried with $\mathrm{MgSO}_{4}$, filtered and concentrated. The yellow oil was 
purified by flash chromatography ( $100 \%$ pentane, $\mathrm{R}_{\mathrm{f}}=0.37$ in $100 \%$ pentane) yielding $55.6 \mathrm{mg}(86 \%)$ of 6a. ${ }^{1} \mathrm{H}$ NMR $\left(500 \mathrm{MHz}, \mathrm{CDCl}_{3}\right) \delta 0.06(\mathrm{~s}, 6 \mathrm{H}), 0.90(\mathrm{~m}, 12 \mathrm{H}), 1.25-$ $1.40(\mathrm{~m}, 6 \mathrm{H}), 2.05(\mathrm{q}, \mathrm{J}=6.6 \mathrm{~Hz}, 2 \mathrm{H}), 2.79(\mathrm{t}, \mathrm{J}=6.9 \mathrm{~Hz}, 2 \mathrm{H}), 3.60(\mathrm{~s}, 2 \mathrm{H}), 5.30-5.45$ $(\mathrm{m}, 4 \mathrm{H}) .{ }^{13} \mathrm{C}$ NMR $\left(125.6 \mathrm{MHz}, \mathrm{CDCl}_{3}\right) \delta-5.0,15.0,18.0,22.8,25.9,26.1,27.4,29.5$, 31.7, 63.0, 125.9, 127.9, 130.3, 130.6. IR: 3012, 2958, 2929, 2858, 1654, 1471. HRMS (CI, M+1) for $\mathrm{C}_{18} \mathrm{H}_{35} \mathrm{D}_{2} \mathrm{OSi}$ calcd 299.2739, found 299.2738.

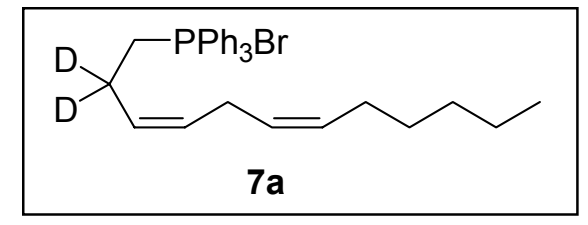

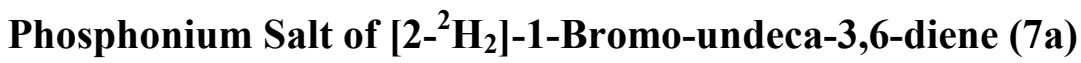

A flask charged with $6 \mathbf{6}(260.4 \mathrm{mg}, 0.87 \mathrm{mmol})$ was purged with argon. $\mathrm{CH}_{2} \mathrm{Cl}_{2}(10 \mathrm{~mL})$ was added followed by a $1.0 \mathrm{M}$ solution of tetrabutylammonium fluoride in THF (1.4 $\mathrm{mL}, 1.40 \mathrm{mmol}$ ). The reaction was stirred at room temperature for $36 \mathrm{~h}$, and then the solvent was removed under vacuum. The resulting oil was purified by flash chromatography (20\% ether in pentane, $\mathrm{R}_{\mathrm{f}}=0.43$ in $20 \%$ EtOAc in Hex) yielding 148.7 $\mathrm{mg}(93 \%)$ of $\left[2-{ }^{2} \mathrm{H}_{2}\right]$-undeca-3,6-dien-1-ol as a clear oil. ${ }^{1} \mathrm{H} \mathrm{NMR}\left(500 \mathrm{MHz}, \mathrm{CDCl}_{3}\right) \delta$ $0.90(\mathrm{t}, \mathrm{J}=6.8 \mathrm{~Hz}, 3 \mathrm{H}), 1.25-1.40(\mathrm{~m}, 6 \mathrm{H}), 2.07(\mathrm{q}, \mathrm{J}=7.0 \mathrm{~Hz}, 2 \mathrm{H}), 2.84(\mathrm{t}, \mathrm{J}=7.1 \mathrm{~Hz}$, $2 \mathrm{H}), 3.66(\mathrm{~s}, 2 \mathrm{H}), 5.31-5.45(\mathrm{~m}, 3 \mathrm{H}), 5.53-5.58(\mathrm{~m}, 1 \mathrm{H}) .{ }^{13} \mathrm{C} \mathrm{NMR}\left(125.6 \mathrm{MHz}, \mathrm{CDCl}_{3}\right) \delta$ $14.3\left(\mathrm{CH}_{3}\right), 19.4\left(\mathrm{CH}_{2}\right), 22.6\left(\mathrm{CH}_{2}\right), 27.0\left(\mathrm{CH}_{2}\right), 30.8\left(\mathrm{CH}_{2}\right), 30.8\left(\mathrm{CH}_{2}\right), 35.7\left(\mathrm{CH}_{2}\right), 61.5$ $\left(\mathrm{CH}_{2}\right), 127.9(\mathrm{CH}), 129.9(\mathrm{CH}), 133.8(\mathrm{CH}), 135.8(\mathrm{CH})$. IR: 3339 (b), 3011, 2958, 2927, 2858, 1652, 1466. HRMS (EI, M+) for $\mathrm{C}_{12} \mathrm{H}_{20} \mathrm{D}_{2} \mathrm{O}$ calcd 184.1796, found 184.1801

A flask was charged with triphenylphosphine $(231.0 \mathrm{mg}, 0.86 \mathrm{mmol})$. After purging with argon, $10 \mathrm{~mL}$ of $\mathrm{CH}_{2} \mathrm{Cl}_{2}$ was added. After cooling to $-10{ }^{\circ} \mathrm{C}$ bromine $(45 \mu \mathrm{L}, 0.86 \mathrm{mmol})$ was added to the solution and the reaction was stirred for $20 \mathrm{~min}$. Pyridine $(70 \mu \mathrm{L}, 0.86$ mmol) was added and stirred for an additional $20 \mathrm{~min}$. Then $\left[2-{ }^{2} \mathrm{H}_{2}\right]$-undeca-3,6-dien-1ol $(131.6 \mathrm{mg}, 0.71 \mathrm{mmol})$ was added in $2 \mathrm{~mL}$ of $\mathrm{CH}_{2} \mathrm{Cl}_{2}$. After $1.5 \mathrm{~h}$, the reaction was quenched with water and extracted with $\mathrm{CH}_{2} \mathrm{Cl}_{2}(3 \times 30 \mathrm{~mL})$. The combined organic layer was washed with $\mathrm{H}_{2} \mathrm{O}$, dried with $\mathrm{MgSO}_{4}$, filtered and concentrated. The resulting yellow oil was purified by flash chromatography $\left(100 \%\right.$ pentane, $\mathrm{R}_{\mathrm{f}}=0.53$ in $100 \%$ pentane) yielding $179.5 \mathrm{mg}(100 \%)$ of $\left[2-{ }^{2} \mathrm{H}_{2}\right]-1$-bromo-undeca-3,6-diene as a clear oil. ${ }^{1} \mathrm{H}$ NMR $\left(500 \mathrm{MHz}, \mathrm{CDCl}_{3}\right) \delta 0.89(\mathrm{t}, \mathrm{J}=7.0 \mathrm{~Hz}, 3 \mathrm{H}), 1.25-1.40(\mathrm{~m}, 6 \mathrm{H}), 2.04(\mathrm{q}, \mathrm{J}=$ $6.6 \mathrm{~Hz}, 2 \mathrm{H}), 2.79(\mathrm{t}, \mathrm{J}=7.1 \mathrm{~Hz}, 2 \mathrm{H}), 3.35(\mathrm{~s}, 2 \mathrm{H}), 5.28-5.45(\mathrm{~m}, 3 \mathrm{H}), 5.48-5.56(\mathrm{~m}, 1 \mathrm{H})$. ${ }^{13} \mathrm{C}$ NMR $\left(125.6 \mathrm{MHz}, \mathrm{CDCl}_{3}\right) \delta 14.2\left(\mathrm{CH}_{3}\right), 22.7\left(\mathrm{CH}_{2}\right), 26.0\left(\mathrm{CH}_{2}\right), 27.4\left(\mathrm{CH}_{2}\right), 29.5$ $\left(\mathrm{CH}_{2}\right), 29.9\left(\mathrm{CH}_{2}\right), 31.7\left(\mathrm{CH}_{2}\right), 126.1(\mathrm{CH}), 127.3(\mathrm{CH}), 131.0(\mathrm{CH}), 131.5(\mathrm{CH})$. LRMS (CI, M+1) 247.

A flask charged with $\left[2-{ }^{2} \mathrm{H}_{2}\right]-1$-bromo-undeca-3,6-diene $(158.9 \mathrm{mg}, 0.64 \mathrm{mmol})$ was purged with argon and acetonitrile $(3 \mathrm{~mL})$ was added. Triphenylphosphine $(253.2 \mathrm{mg}$, $0.96 \mathrm{mmol}$ ) was added and the solution was refluxed for $36 \mathrm{~h}$. After cooling to room temperature, the reaction was poured onto a column impregnated with $1 / 1$ pentane/ether. After elution of excess triphenylphosphine, the product was eluted using $10 \%$ methanol in $\mathrm{CH}_{2} \mathrm{Cl}_{2}$ as mobile phase. Yield $309.9 \mathrm{mg}(95 \%)$ of $7 \mathbf{a}$ as a gummy clear oil. ${ }^{1} \mathrm{H} \mathrm{NMR}$ $\left(400 \mathrm{MHz}, \mathrm{CDCl}_{3}\right) \delta 0.86(\mathrm{t}, \mathrm{J}=7.1 \mathrm{~Hz}, 2 \mathrm{H}), 1.14-1.32(\mathrm{~m}, 6 \mathrm{H}), 1.86(\mathrm{q}, \mathrm{J}=7.2 \mathrm{~Hz}, 2 \mathrm{H})$, $2.52(\mathrm{t}, \mathrm{J}=7.3 \mathrm{~Hz}, 2 \mathrm{H}), 3.99(\mathrm{~d}, \mathrm{~J}=12.3 \mathrm{~Hz}, 2 \mathrm{H}), 5.10-5.17(\mathrm{~m}, 1 \mathrm{H}), 5.27-5.40(\mathrm{~m}, 2 \mathrm{H})$, 
5.58-5.63 (m, 1H), 7.67-7.73 (m, 6H), 7.76-7.82 (m, 3H), 7.85-7.94 (m 6H). ${ }^{31} \mathrm{P}$ NMR $\left(161.9 \mathrm{MHz}, \mathrm{CDCl}_{3}\right) \delta 25.6(\mathrm{~s})$. HRMS (FAB, M-Br) for $\mathrm{C}_{30} \mathrm{H}_{34} \mathrm{D}_{2} \mathrm{P}$ calcd 429.2680, found 429.2662 .

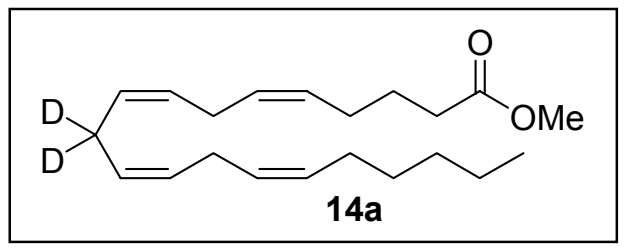

\section{$\left[10,10-{ }^{2} \mathrm{H}_{2}\right]-$ Methyl arachidonate (14a)}

Triphenylphosphonium salt 7a $(302.1 \mathrm{mg}, 0.59 \mathrm{mmol})$ was dried three times by azeotropic evaporation with anhydrous benzene. THF $(10 \mathrm{~mL})$ was added and the solution was cooled to $-78^{\circ} \mathrm{C}$. A solution of $1.0 \mathrm{NaHMDS}$ in THF (500 $\left.\mu \mathrm{L}, 0.50 \mathrm{mmol}\right)$ was added and the reaction was stirred for $45 \mathrm{~min}$. The solution was cooled to $-90{ }^{\circ} \mathrm{C}$, and aldehyde $13(198.2 \mathrm{mg}, 1.16 \mathrm{mmol})$ in $2 \mathrm{~mL}$ of THF was added dropwise. The reaction was stirred at $-90{ }^{\circ} \mathrm{C}$ for $5 \mathrm{~h}$, and then slowly warmed to room temperature overnight. The reaction was quenched with water and extracted with ether $(4 \times 40 \mathrm{~mL})$. The combined organic layers were washed with water $(1 \times 30 \mathrm{~mL})$, dried with $\mathrm{MgSO}_{4}$, filtered and concentrated. The resulting oil was purified by flash chromatography $(5 \%$ ether in pentane, $\mathrm{R}_{\mathrm{f}}=0.50$ in $5 \%$ EtOAc in Hex) yielding $118.0 \mathrm{mg}(77 \%)$ of $\mathbf{1 4 a}$ as a clear oil. ${ }^{1} \mathrm{H}$ NMR $\left(400 \mathrm{MHz}, \mathrm{CDCl}_{3}\right) \delta 0.88(\mathrm{t}, \mathrm{J}=6.8 \mathrm{~Hz}, 3 \mathrm{H}), 1.23-1.40(\mathrm{~m}, 6 \mathrm{H}), 1.70$ (pentet, J = 7.4 Hz, 2H), $2.05(\mathrm{q}, \mathrm{J}=7.0 \mathrm{~Hz}, 2 \mathrm{H}), 2.11(\mathrm{q}, \mathrm{J}=7.1 \mathrm{~Hz}, 2 \mathrm{H}), 2.32(\mathrm{t}, \mathrm{J}=7.5$ $\mathrm{Hz}, 2 \mathrm{H}), 2.77-2.83(\mathrm{~m}, 4 \mathrm{H}), 3.66(\mathrm{~s}, 3 \mathrm{H}), 5.28-5.43(\mathrm{~m}, 8 \mathrm{H}) .{ }^{13} \mathrm{C} \mathrm{NMR}(100.6 \mathrm{MHz}$, $\left.\mathrm{CDCl}_{3}\right), \delta 14.3\left(\mathrm{CH}_{3}\right), 22.8\left(\mathrm{CH}_{2}\right), 25.0\left(\mathrm{CH}_{2}\right), 25.8\left(\mathrm{CH}_{2}\right), 25.9\left(\mathrm{CH}_{2}\right), 26.8\left(\mathrm{CH}_{2}\right), 27.4$ $\left(\mathrm{CH}_{2}\right), 29.6\left(\mathrm{CH}_{2}\right), 31.7\left(\mathrm{CH}_{2}\right), 33.4\left(\mathrm{CH}_{2}\right), 51.7\left(\mathrm{CH}_{3}\right), 127.8(\mathrm{CH}), 128.0(\mathrm{CH}), 128.3$ $(\mathrm{CH}), 128.4(\mathrm{CH}), 128.8(\mathrm{CH}), 129.1(\mathrm{CH}), 129.2(\mathrm{CH}), 130.7(\mathrm{CH}), 174.3(\mathrm{Cq})$. HRMS (EI, $\mathrm{M}+$ ) for $\mathrm{C}_{21} \mathrm{H}_{32} \mathrm{D}_{2} \mathrm{O}_{2}$ calcd 320.2684 , found 320.2690 .

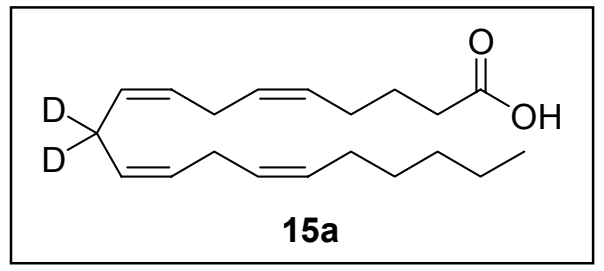

\section{[10,10- $\left.{ }^{2} \mathrm{H}_{2}\right]$-Arachidonic Acid (15a)}

A flask was charged with 14a (34.1 $\mathrm{mg}, 0.11 \mathrm{mmol})$ and flushed with argon. THF $(4 \mathrm{~mL})$ was added and the solution was cooled to $0{ }^{\circ} \mathrm{C}$. A solution of $1.0 \mathrm{M} \mathrm{LiOH}(2.0 \mathrm{~mL})$ was added dropwise and the reaction was stirred for $1 \mathrm{~h}$, then slowly warmed to room temperature. After $16 \mathrm{~h}$, the $\mathrm{pH}$ of the reaction was adjusted to $\mathrm{pH}=1$ using $1 \mathrm{M} \mathrm{HCl}$ and the solution was extracted with ether $(5 \times 20 \mathrm{~mL})$. The combined organic layers were washed with water $(1 \times 10 \mathrm{~mL})$, dried with $\mathrm{MgSO}_{4}$, filtered and concentrated. The resulting oil was purified by flash chromatography (15\% ether in pentane to $30 \%$ ether in pentane, $\mathrm{R}_{\mathrm{f}}=0.20$ in $10 \%$ EtOAc in Hex) yielding $30.7 \mathrm{mg}(93 \%)$ of $\mathbf{1 5 a}$ as a clear oil. ${ }^{1} \mathrm{H}$ NMR $\left(400 \mathrm{MHz}, \mathrm{CDCl}_{3}\right) \delta 0.88(\mathrm{t}, \mathrm{J}=7.0 \mathrm{~Hz}, 3 \mathrm{H}), 1.24-1.40(\mathrm{~m}, 6 \mathrm{H}), 1.72$ (pentet, $\mathrm{J}$ $=7.4 \mathrm{~Hz}, 2 \mathrm{H}), 2.05(\mathrm{q}, \mathrm{J}=7.0 \mathrm{~Hz}, 2 \mathrm{H}), 2.13(\mathrm{q}, \mathrm{J}=7.1 \mathrm{~Hz}, 2 \mathrm{H}), 2.37(\mathrm{t}, \mathrm{J}=7.5 \mathrm{~Hz}, 2 \mathrm{H})$, 
2.78-2.83 (m, 4H), 5.30-5.45 (m, 8H). $\left.{ }^{13} \mathrm{C} \mathrm{NMR} \mathrm{(100.6} \mathrm{MHz,} \mathrm{CDCl}_{3}\right) \delta 14.3\left(\mathrm{CH}_{3}\right), 22.8$ $\left(\mathrm{CH}_{2}\right), 24.7\left(\mathrm{CH}_{2}\right), 25.8\left(\mathrm{CH}_{2}\right), 25.9\left(\mathrm{CH}_{2}\right), 26.7\left(\mathrm{CH}_{2}\right), 27.4\left(\mathrm{CH}_{2}\right), 29.6\left(\mathrm{CH}_{2}\right), 31.8$ $\left(\mathrm{CH}_{2}\right), 33.6\left(\mathrm{CH}_{2}\right), 127.8(\mathrm{CH}), 128.0(\mathrm{CH}), 128.4(\mathrm{CH}), 128.8(\mathrm{CH}), 129.0(\mathrm{CH}), 129.3$ $(\mathrm{CH}), 130.7(\mathrm{CH}), 179.9(\mathrm{Cq})$. HRMS (EI, M+) for $\mathrm{C}_{20} \mathrm{H}_{20} \mathrm{D}_{2} \mathrm{O}_{2}$ calcd 307.2606, found 307.2625 .

\section{HPLC purification of $\left[10,10-{ }^{2} \mathrm{H}_{2}\right]$-Arachidonic Acid}

The final arachidonic acid product was purified by HPLC using a Microsorb C18 column. Mobile Phase A: 90/10/0.1 MeCN/ $\mathrm{H}_{2} \mathrm{O} / \mathrm{AcOH}$, Mobile Phase B: 50/50/0.1 $\mathrm{ACN} / \mathrm{H}_{2} \mathrm{O} / \mathrm{AcOH}$. Isocratic method using $30 \% \mathrm{MPA} / 70 \% \mathrm{MPB}$. Flow rate : $1 \mathrm{~mL} / \mathrm{min}$. Detection: $210 \mathrm{~nm}$.

\section{$\left[13,13-{ }^{2} \mathbf{H}_{2}\right]$-Arachidonic Acid}

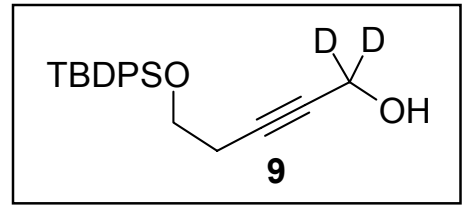

\section{5-(tert-Butyl-diphenyl-silanyloxy)-1,1-d 2 -pent-2-yn-1-ol (9)}

tert-Butyl-but-3-ynyloxy-diphenyl-silane $8(6 \mathrm{~g}, 19.5 \mathrm{mmol})$ was added dropwise to a solution of $1 \mathrm{M} \mathrm{EtMgBr}(29 \mathrm{~mL}, 29 \mathrm{mmol})$ in THF at room temperature. After refluxing for $2 \mathrm{~h}$, the reaction mixture was cooled to $0{ }^{\circ} \mathrm{C}$ and $\left(\mathrm{CD}_{2} \mathrm{O}\right)_{\mathrm{n}}(836 \mathrm{mg})$ was added. The reaction was refluxed for $3 \mathrm{~h}$ and cooled to room temperature. Saturated ammonium chloride solution was added to quench the reaction. After extraction with $\mathrm{Et}_{2} \mathrm{O}(3 \times 150$ $\mathrm{mL}$ ), the combined organic layers were dried with $\mathrm{NaSO}_{4}$, concentrated under reduced pressure, and the crude product was purified by flash chromatography $\left(\mathrm{R}_{\mathrm{f}} 0.2\right.$, hexane:EtOAc $=100: 5)$ to provide 5-(tert-butyl-diphenyl-silanyloxy)-1,1- $d_{2}$-pent-2-yn1-ol $9(5.7 \mathrm{~g}, 91 \%)$ as a colorless oil. ${ }^{1} \mathrm{H}$ NMR $\left(500 \mathrm{MHz}, \mathrm{CDCl}_{3}\right) \delta 7.76-7.43(10 \mathrm{H}, \mathrm{m})$, $3.83(2 \mathrm{H}, \mathrm{t}, \mathrm{J}=7.1 \mathrm{~Hz}), 2.53(2 \mathrm{H}, \mathrm{t}, \mathrm{J}=7.0 \mathrm{~Hz}), 2.0(1 \mathrm{H}, \mathrm{br}), 1.13(9 \mathrm{H}, \mathrm{s}) ;{ }^{13} \mathrm{C}$ NMR $(125$ $\left.\mathrm{MHz}, \mathrm{CDCl}_{3}\right) \delta 135.8,133.8,130.0,128.0,83.5,79.8,62.7,27.1,23.1,19.5$.

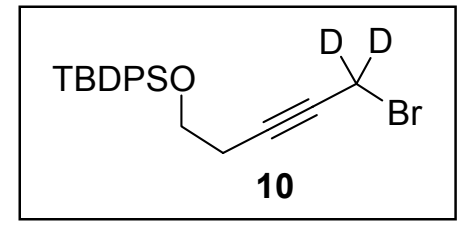

(5-Bromo-5,5- $\boldsymbol{d}_{\mathbf{2}}$-pent-3-ynyloxy)-tert-butyl-diphenyl-silane (10) A solution of triphenylphosphine $(4.11 \mathrm{~g}, 15.6 \mathrm{mmol})$ in $\mathrm{CH}_{2} \mathrm{Cl}_{2}(40 \mathrm{~mL})$ was added to a solution of 5-(tert-butyl-diphenyl-silanyloxy)-1,1- $d_{2}$-pent-2-yn-1-ol $9(3.4 \mathrm{~g}, 10 \mathrm{mmol})$ and carbon tetrabromide $(4.74 \mathrm{~g}, 142 \mathrm{mmol})$ in $21 \mathrm{~mL}$ of $\mathrm{CH}_{2} \mathrm{Cl}_{2}$-pyridine (20:1). The reaction mixture was stirred for $4 \mathrm{~h}$ at $25{ }^{\circ} \mathrm{C}$ then filtered through a short silica gel column to afford (5-bromo-5,5- $d_{2}$-pent-3-ynyloxy)-tert-butyl-diphenyl-silane $\mathbf{1 0}$ ( $2.8 \mathrm{~g}$, $69 \%)$ as a colorless oil . ${ }^{1} \mathrm{H}$ NMR $\left(400 \mathrm{MHz}, \mathrm{CDCl}_{3}\right) \delta 7.76-7.43(10 \mathrm{H}, \mathrm{m}), 3.83(2 \mathrm{H}, \mathrm{t}$, 
$\mathrm{J}=7.0 \mathrm{~Hz}), 2.33(2 \mathrm{H}, \mathrm{t}, \mathrm{J}=7.1 \mathrm{~Hz}), 1.13(9 \mathrm{H}, \mathrm{s}) ;{ }^{13} \mathrm{C} \mathrm{NMR}\left(125 \mathrm{MHz}, \mathrm{CDCl}_{3}\right) \delta 135.83$, 133.73, 129.96, 127.96, 63.13, 62.29, 30.73, 26.98, 23.30, 19.57, 19.43; EIMS (m/z) 403 $\left(\mathrm{M}^{+}\right)$; HRMS (EI) calcd. for $\mathrm{C}_{21} \mathrm{H}_{24} \mathrm{D}_{2} \mathrm{OSiBr} 403.106184$ found 403.105021 .

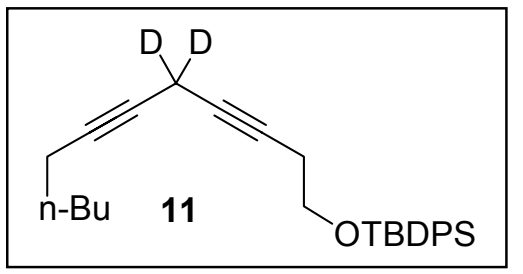

tert-Butyl-(5,5- $\boldsymbol{d}_{\mathbf{2}}$-dodeca-3,6-diynyloxy)-diphenyl-silane (11)

1-Heptyne $(0.88 \mathrm{~mL}, 6.7 \mathrm{mmol})$ was added to a $1 \mathrm{M}$ solution of EtMgBr in THF $(8.5 \mathrm{~mL}$, $8.5 \mathrm{mmol}$ ) at room temperature, followed by refluxing for $1 \mathrm{~h}$. After the reaction was cooled to room temperature, copper(I) bromide $(20 \mathrm{mg}$ ) was added, followed by (5bromo-5,5- $d_{2}$-pent-3-ynyloxy)-tert-butyl-diphenyl-silane 10 in THF $(1 \mathrm{~mL})$. The reaction mixture was refluxed overnight. Saturated ammonium chloride solution was added to quench the reaction. After extraction with diethyl ether, the combined organic layers were dried with $\mathrm{NaSO}_{4}$, concentrated under reduced pressure, and the crude product was purified by flash chromatography $\left(\mathrm{R}_{\mathrm{f}} 0.3\right.$, hexane:EtOAc $\left.=100: 1\right)$ providing tert-butyl(5,5- $d_{2}$-dodeca-3,6-diynyloxy)-diphenyl-silane $11(1.7 \mathrm{~g}, 86 \%) .{ }^{1} \mathrm{H}$ NMR $(500 \mathrm{MHz}$, $\left.\mathrm{CDCl}_{3}\right) \delta$ 7.71-7.09 $(4 \mathrm{H}, \mathrm{m}), 7.43-7.40(6 \mathrm{H}, \mathrm{m}), 3.79-3.75(2 \mathrm{H}, \mathrm{m}), 3.52-3.48(2 \mathrm{H}, \mathrm{m})$, $2.46(2 \mathrm{H}, \mathrm{t}, \mathrm{J}=7.1 \mathrm{~Hz}), 2.25(2 \mathrm{H}, \mathrm{t}, \mathrm{J}=7.0 \mathrm{~Hz}), 2.15(2 \mathrm{H}, \mathrm{t}, \mathrm{J}=7.0 \mathrm{~Hz}), 1.52-1.45(2 \mathrm{H}, \mathrm{m})$, 1.36-1.19 (6H, m), $1.34(9 \mathrm{H}, \mathrm{s}), 0.90(3 \mathrm{H}, \mathrm{t}, \mathrm{J}=7.0 \mathrm{~Hz}) ; \mathrm{MS}(\mathrm{m} / \mathrm{z}) 361\left(\mathrm{M}^{+}-57\right)$; HRMS (EI) calcd. for $\mathrm{C}_{24} \mathrm{H}_{25} \mathrm{D}_{2} \mathrm{OSi} 361.195673$ found 361.195317 .

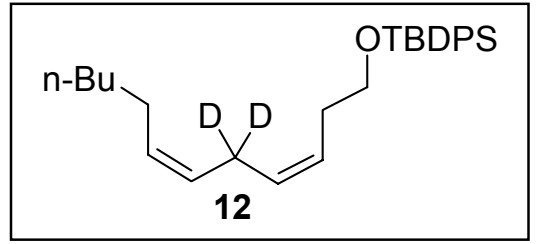

tert-Butyl-(5,5- $d_{2}$-dodeca-3,6-dienyloxy)-diphenyl-silane (12)

Under $\mathrm{Ar}, \mathrm{Ni}(\mathrm{OAc})_{2}-\mathrm{H}_{2} \mathrm{O}(102 \mathrm{mg})$ in $11 \mathrm{~mL}$ of ethanol was reduced with a $1 \mathrm{M}$ solution of $\mathrm{NaBH}_{4}$ in a mixture of $2 \mathrm{M}$ aqueous $\mathrm{NaOH}$ and ethanol $(0.41 \mathrm{~mL})$. Ethylenediamine $(0.1 \mathrm{~mL})$ was added, followed by tert-butyl-(5,5- $d_{2}$-dodeca-3,6-diynyloxy)-diphenylsilane $11(1.7 \mathrm{~g}, 4 \mathrm{mmol})$ in $11 \mathrm{~mL}$ of ethanol. The reaction was stirred under hydrogen at room temperature overnight and the solvent was evaporated under reduced pressure. The crude product was purified by silica gel flash chromatography $\left(\mathrm{R}_{\mathrm{f}} 0.1,100 \%\right.$ hexane), and tert-butyl-(5,5- $d_{2}$-dodeca-3,6-dienyloxy)-diphenyl-silane $\mathbf{1 2}$ was obtained as a colorless oil (1.0 g, 63\%). ${ }^{1} \mathrm{H}$ NMR (500 MHz, $\left.\mathrm{CDCl}_{3}\right) \delta$ 7.77-7.43 (10H, m), 5.51-5.35 (4H, m), $3.75(2 \mathrm{H}, \mathrm{t}, \mathrm{J}=6.8 \mathrm{~Hz}), 2.43(2 \mathrm{H}, \mathrm{d}-\mathrm{t}, \mathrm{J}=7.0 \mathrm{~Hz}, \mathrm{~J}=7.0 \mathrm{~Hz}), 2.10(2 \mathrm{H}, \mathrm{d}-\mathrm{t}-\mathrm{d}, \mathrm{J}=6.4 \mathrm{~Hz}$, $\mathrm{J}=6.4 \mathrm{~Hz}, \mathrm{~J}=1.3 \mathrm{~Hz}), 1.41-1.34(6 \mathrm{H}, \mathrm{m}), 1.3(9 \mathrm{H}, \mathrm{s}), 0.96(3 \mathrm{H}, \mathrm{t}, \mathrm{J}=6.2 \mathrm{~Hz}) ;{ }^{13} \mathrm{C} \mathrm{NMR}$ $\left(125 \mathrm{MHz} \mathrm{CDCl}_{3}\right) \delta 135.87,134.25,130.63,130.19,129.84,127.90,126.23,63.89$, 31.79, 29.62, 27.48, 27.11, 22.88, 19.46, 14.36; EIMS (m/z) $365\left(\mathrm{M}^{+}-57\right)$; HRMS (EI) calcd. for $\mathrm{C}_{24} \mathrm{H}_{29} \mathrm{D}_{2} \mathrm{OSi}\left(\mathrm{M}^{+}-57\right) 365.226973$ found 365.22668 . 


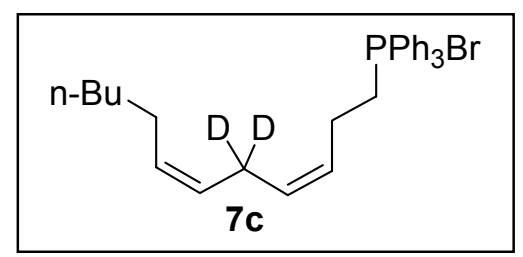

\section{(5,5- $\boldsymbol{d}_{\mathbf{2}}$-Dodeca-3,6-dienyl)-triphenyl-phosphonium bromide (7c)}

A $1 \mathrm{M}$ solution of TBAF in THF $(3.6 \mathrm{~mL}, 3.6 \mathrm{mmol})$ was added to tert-butyl- $\left(5,5-d_{2}-\right.$ dodeca-3,6-dienyloxy)-diphenyl-silane $12(385 \mathrm{mg}, 0.91 \mathrm{mmol})$ in $6 \mathrm{~mL}$ of THF and 0.54 $\mathrm{mL}$ of acetic acid at room temperature and the reaction was stirred overnight. The solvent was evaporated under reduced pressure and the resulting material was purified by flash chromatography on a silica gel column $\left(\mathrm{R}_{\mathrm{f}} 0.2\right.$, hexane:EtOAc $\left.=90: 10\right)$ to give 5,5- $d_{2}$ dodeca-3,6-dien-1-ol (170 mg, 100\%) as a colorless oil. ${ }^{1} \mathrm{H}$ NMR $\left(500 \mathrm{MHz}, \mathrm{CDCl}_{3}\right) \delta$ 5.53-5.31 (4H, m), $3.65(2 \mathrm{H} \mathrm{t}, \mathrm{J}=7.0 \mathrm{~Hz}), 2.38-2.33(2 \mathrm{H}, \mathrm{m}), 2.05(2 \mathrm{H}, \mathrm{d}-\mathrm{t}-\mathrm{d}, \mathrm{J}=7.0 \mathrm{~Hz}$, $\mathrm{J}=7.0 \mathrm{~Hz}, \mathrm{~J}=1.5 \mathrm{~Hz}), 1.87(1 \mathrm{H}, \mathrm{br}), 1.36-1.26(6 \mathrm{H}, \mathrm{m}), 0.89(3 \mathrm{H}, \mathrm{t}, \mathrm{J}=7.2 \mathrm{~Hz}) ;{ }^{13} \mathrm{C} \mathrm{NMR}$ $\left(125 \mathrm{MHz}, \mathrm{CDCl}_{3}\right) \delta 131.53,130.87,127.51,125.62,62.39,31.70,31.00,29.51,27.43$, 22.77, 14.25; EIMS (m/z) $184\left(\mathrm{M}^{+}\right)$; HRMS (EI) calcd. for $\mathrm{C}_{12} \mathrm{H}_{20} \mathrm{D}_{2} \mathrm{O} 184.179619$ found 184.17953.

To a solution of 5,5- $d_{2}$-dodeca-3,6-dien-1-ol (160 mg, $\left.0.87 \mathrm{mmol}\right)$ and pyridine $(116 \mathrm{mg})$ in acetonitrile $(3 \mathrm{~mL})$ was added $\mathrm{PPh}_{3} \mathrm{Br}_{2}(510 \mathrm{mg}, 1.29 \mathrm{mmol})$ at $0{ }^{\circ} \mathrm{C}$. The reaction mixture was stirred at room temperature for $1 \mathrm{~h}$ and then filtered through a short plug of silica gel $\left(\mathrm{R}_{\mathrm{f}} 0.6,100 \%\right.$ hexane $)$ to provide 1-bromo-5,5- $d_{2}$-dodeca-3,6-diene (180 $\mathrm{mg}$, $84 \%)$ as a colorless oil. ${ }^{1} \mathrm{H}$ NMR $\left(500 \mathrm{MHz}, \mathrm{CDCl}_{3}\right) \delta 5.52-5.30(4 \mathrm{H}, \mathrm{m}), 3.37(2 \mathrm{H}, \mathrm{t}$, $\mathrm{J}=7.2 \mathrm{~Hz}), 2.65(2 \mathrm{H}, \mathrm{d}-\mathrm{t}-\mathrm{d}, \mathrm{J}=7.0 \mathrm{~Hz}, \mathrm{~J}=7.0 \mathrm{~Hz}, \mathrm{~J}=1.5 \mathrm{~Hz}), 1.36-1.28(6 \mathrm{H}, \mathrm{m}), 0.89(3 \mathrm{H}, \mathrm{t}$, $\mathrm{J}=7.2 \mathrm{~Hz}) ;{ }^{13} \mathrm{C}$ NMR $\left(125 \mathrm{MHz}, \mathrm{CDCl}_{3}\right) \delta 131.41,131.03,127.19,126.32,32.55,31.74$, 31.06, 29.52, 27.47, 22.80, 14.29; EIMS (m/z) $246\left(\mathrm{M}^{+}\right), 248\left(\mathrm{M}^{+}\right)$; HRMS (EI) calcd. for $\mathrm{C}_{12} \mathrm{H}_{19} \mathrm{D}_{2} \mathrm{Br} 248.093169$ found 248.093169.

A mixture of 1-bromo-5,5- $d_{2}$-dodeca-3,6-diene $(170 \mathrm{mg}, \quad 0.7 \mathrm{mmol})$ and triphenylphosphine $(367 \mathrm{mg}, 1.4 \mathrm{mmol})$ in acetonitrile $(2 \mathrm{~mL})$ was refluxed for $48 \mathrm{~h}$. The reaction mixture was purified by silica gel flash chromatography $\left(\mathrm{R}_{\mathrm{f}} 0.6, \mathrm{CH}_{2} \mathrm{Cl}_{2}: \mathrm{MeOH}\right.$ $=100: 5)$ to yield the product $7 \mathrm{c}(350 \mathrm{mg}, 100 \%)$ as a yellow foam. ${ }^{1} \mathrm{H}$ NMR $(500 \mathrm{MHz}$, $\left.\mathrm{CDCl}_{3}\right) \delta$ 7.85-7.68 (15H, m), 5.60-5.10 (4H, m), 3.86-3.80 (2H, m), 2.48-2.41 (2H, m), $1.83(2 \mathrm{H}, \mathrm{d}-\mathrm{t}, \mathrm{J}=7.0 \mathrm{~Hz}, \mathrm{~J}=7.0 \mathrm{~Hz}), 1.27-1.15(6 \mathrm{H}, \mathrm{m}), 0.82(3 \mathrm{H}, \mathrm{t}, \mathrm{J}=7.0 \mathrm{~Hz}) ;{ }^{13} \mathrm{C} \mathrm{NMR}$ $\left(125 \mathrm{MHz}, \mathrm{CDCl}_{3}\right) \delta 135.39,135.37,133.96,133.88131 .11,130.84,130.74,130.71$, $126.76,118.68,118.0,31.63,29.35,27.36,23.31,22.94,22.73,20.58,14.29$; FABMS $(\mathrm{m} / \mathrm{z}) 429\left(\mathrm{M}^{+}-\mathrm{Br}\right)$; HRMS (FAB) calcd. for $\mathrm{C}_{30} \mathrm{H}_{34} \mathrm{D}_{2} \mathrm{P}\left(\mathrm{M}^{+}-\mathrm{Br}\right) 429.268200$ found 429.268018 . 


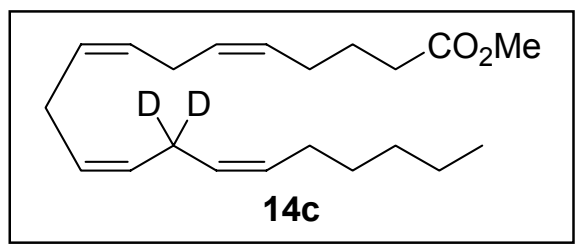

\section{Methyl 13,13- $d_{2}$-arachidonate (14c)}

(5,5- $d_{2}$-Dodeca-3,6-dienyl)-triphenyl-phosphonium bromide 7c (200 mg, $\left.0.39 \mathrm{mmol}\right)$ was dried under vacuum for $30 \mathrm{~min}$ and THF $(5 \mathrm{~mL})$ was added, followed by a $1 \mathrm{M}$ solution of sodium bis(trimethylsilyl)amide in THF $(0.38 \mathrm{~mL}, 0.38 \mathrm{mmol})$ at $-70{ }^{\circ} \mathrm{C}$. The orange solution of the ylide was stirred at $-70{ }^{\circ} \mathrm{C}$ for $1 \mathrm{~h}$. The mixture was cooled to $-100{ }^{\circ} \mathrm{C}$, and a solution of 8-oxo-oct-5-enoic acid methyl ester 13 (95 mg, $0.54 \mathrm{mmol})$ in THF (2 $\mathrm{mL}$ ) was added dropwise. After $0.5 \mathrm{~h}$ at $-100{ }^{\circ} \mathrm{C}$, the reaction was allowed to warm to room temperature over $5 \mathrm{~h}$. Saturated aqueous sodium bicarbonate was added and the aqueous phase was extracted with $\mathrm{Et}_{2} \mathrm{O}(3 \times 100 \mathrm{~mL})$. The combined organic phases were washed with brine and dried over anhydrous $\mathrm{NaSO}_{4}$, concentrated, and purified by silica gel chromatography $\left(\mathrm{R}_{\mathrm{f}} 0.5\right.$, hexane:EtOAc $\left.=100: 5\right)$ to provide methyl $13,13-d_{2}$ arachidonate $14 \mathrm{c}(75 \mathrm{mg}, 61 \%)$ as a colorless oil. ${ }^{1} \mathrm{H} \mathrm{NMR}\left(500 \mathrm{MHz}, \mathrm{CDCl}_{3}\right) \delta 5.44-$ $5.33(8 \mathrm{H}, \mathrm{m}), 3.69(3 \mathrm{H}, \mathrm{s}), 2.86-2.81(4 \mathrm{H}, \mathrm{m}), 2.34(2 \mathrm{H}, \mathrm{t}, \mathrm{J}=7.4 \mathrm{~Hz}), 2.25(2 \mathrm{H}, \mathrm{d}-\mathrm{t}, \mathrm{J}=7.0$ $\mathrm{Hz}, \mathrm{J}=7.0 \mathrm{~Hz}), 2.06(2 \mathrm{H}, \mathrm{d}-\mathrm{t}, \mathrm{J}=7.0 \mathrm{~Hz}, \mathrm{~J}=7.0 \mathrm{~Hz}), 1.72(2 \mathrm{H}$, quin, $\mathrm{J}=7.0 \mathrm{~Hz}), 1.39-1.24$ $(6 \mathrm{H}, \mathrm{m}), 0.90(3 \mathrm{H}, \mathrm{t}, \mathrm{J}=7.0 \mathrm{~Hz}) ;{ }^{13} \mathrm{C} \mathrm{NMR}\left(125 \mathrm{MHz}, \mathrm{CDCl}_{3}\right) \delta 174.29,130.74,129.15$, $129.10,128.70,128.44,128.38,128.12,127.66,51.70,33.62,31.73,29.55,27.44,26.75$, 25.15, 24.98, 22.79, 14.28; EIMS (m/z) $320\left(\mathrm{M}^{+}\right)$.

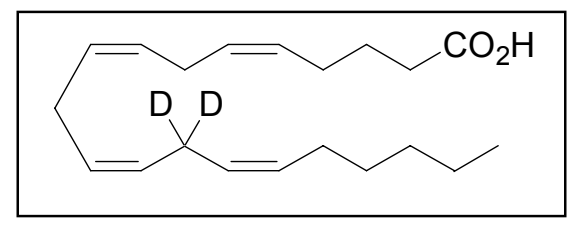

\section{3,13- $d_{2}$-Arachidonic acid (15c)}

Methyl $13,13-d_{2}$-arachidonate $14 \mathbf{c}(26 \mathrm{mg}, 0.08 \mathrm{mmol})$ was dissolved in THF $(1.4 \mathrm{~mL})$, and cooled to $0{ }^{\circ} \mathrm{C}$. A $1 \mathrm{M}$ solution of aqueous $\mathrm{LiOH}(1.4 \mathrm{~mL}, 1.4 \mathrm{mmol})$ was added. The reaction mixture was stirred for $19 \mathrm{~h}$ at room temperature, acidified to $\mathrm{pH} 1$ with $1 \mathrm{M}$ $\mathrm{HCl}$, saturated with solid $\mathrm{NaCl}$, and extracted with $\mathrm{Et}_{2} \mathrm{O}(3 \times 80 \mathrm{~mL})$. After washing the combined organic phases with brine, drying over anhydrous sodium sulfate, and concentration under reduced pressure, purification by silica gel chromatography $\left(\mathrm{R}_{\mathrm{f}} 0.2\right.$, hexane:EtOAc $=100: 10)$ gave $13,13-d_{2}$-AA 15c $(25 \mathrm{mg}, 100 \%) .{ }^{1} \mathrm{H}$ NMR $(500 \mathrm{MHz}$, $\left.\mathrm{CDCl}_{3}\right) \delta$ 5.46-5.34 (8H, m), 2.87-2.82 (6H, m), $2.39(2 \mathrm{H}, \mathrm{t}, \mathrm{J}=7.5 \mathrm{~Hz}), 2.15(2 \mathrm{H}, \mathrm{d}-\mathrm{t}$, $\mathrm{J}=7.0, \mathrm{~J}=7.0 \mathrm{~Hz}), 2.07(2 \mathrm{H}, \mathrm{d}-\mathrm{t}-\mathrm{d}, \mathrm{J}=7.2 \mathrm{~Hz}, \mathrm{~J}=7.0 \mathrm{~Hz}, \mathrm{~J}=1.5 \mathrm{~Hz}), 1.74(2 \mathrm{H}$, quin, $\mathrm{J}=7.5$ $\mathrm{Hz}), 1.40-1.27(6 \mathrm{H}, \mathrm{m}), 0.91(3 \mathrm{H}, \mathrm{t}, \mathrm{J}=7.0 \mathrm{~Hz})$; EIMS $(\mathrm{m} / \mathrm{z}) 305\left(\mathrm{M}^{+}, 8.82\right) ;{ }^{13} \mathrm{C} \mathrm{NMR}$ $\left(125 \mathrm{MHz}, \mathrm{CDCl}_{3}\right) \delta 179.84,130.77,129.28,128.98,128.73,128.49,128.34,128.12$, $127.67,33.55,31.74,31.73,29.56,27.44,26.66,25.83,24.70,22.80,14.30 ;$ EIMS (m/z) $306\left(\mathrm{M}^{+}-1\right)$; HRMS (ESI) calcd. For $\mathrm{C}_{20} \mathrm{H}_{30} \mathrm{DO}_{2} 306.252784$ found 306.252522. Deuterium content: $95 \% d_{2}, 5 \% d_{1}$ by ESI-MS comparison with unlabeled arachidonic acid. 


\section{$\left[11-{ }^{13} \mathrm{C}\right]$-Arachidonic Acid}

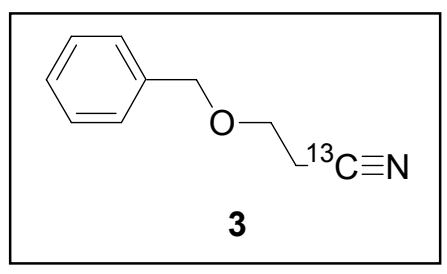

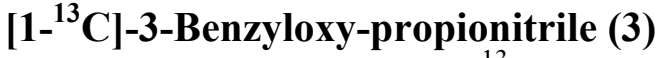

A flask was charged with $\mathrm{K}^{13} \mathrm{CN}(493.5 \mathrm{mg}, 7.57 \mathrm{mmol})$ and $5 \mathrm{~mL}$ of water was added. A $10 \%$ solution of $\mathrm{NaI}$ in ethanol $(10 \mathrm{~mL})$ was added, followed by 2 -chloroethanol $(1.4$ $\mathrm{mL}, 20.9 \mathrm{mmol})$. The reaction was heated at $80{ }^{\circ} \mathrm{C}$ for $7 \mathrm{~h}$ then cooled to room temperature. A negative cyanide test, shown by no change in color upon addition of 1 drop of reaction mixture to $0.5 \mathrm{~mL}$ of a $1 \mathrm{M}$ solution of p-nitrobenzyaldehyde in DMSO, demonstrated the consumption of all cyanide anions. The ethanol was removed under vacuum and the solution purified by silica gel chromatography $\left(50 / 50 \mathrm{Et}_{2} \mathrm{O} /\right.$ pentane to $80 / 20 \mathrm{Et}_{2} \mathrm{O} /$ pentane, $\mathrm{R}_{\mathrm{f}}=0.6$ in $100 \%$ ether) yielding $451.2 \mathrm{mg}(83 \%)$ of 3hydroxypropionitrile as a clear oil. ${ }^{1} \mathrm{H}$ NMR $\left(400 \mathrm{MHz}, \mathrm{CDCl}_{3}\right) \delta 1.80-2.10(\mathrm{~m}, 1 \mathrm{H})$, 2.58-2.64 (m, 2H), $3.89(\mathrm{dq}, \mathrm{J}=0.9 \mathrm{~Hz}, 6.3 \mathrm{~Hz}, 2 \mathrm{H}) .{ }^{13} \mathrm{C} \mathrm{NMR}\left(100.6 \mathrm{MHz}, \mathrm{CDCl}_{3}\right) \delta$ $21.7\left(\mathrm{~d}, \mathrm{~J}=57.3 \mathrm{MHz}, \mathrm{CH}_{2}\right), 58.2\left(\mathrm{~s}, \mathrm{CH}_{2}\right), 118.2$ (s, Cq, enhanced signal). HRMS (EI, $\mathrm{M}+1$ ) for ${ }^{13} \mathrm{CC}_{2} \mathrm{H}_{6} \mathrm{NO}$ calcd 73.0483 , found 73.0488. Spectral data matched that reported in the literature. ${ }^{2}$

A flask was charged with 3-hydroxypropionitrile $(450.2 \mathrm{mg}, 6.33 \mathrm{mmol})$ and purged with Ar. THF $(40 \mathrm{~mL})$ was added, and the reaction was cooled to $0{ }^{\circ} \mathrm{C}$. Sodium hydride (250.2 $\mathrm{mg}, 10.13 \mathrm{mmol}$ ) was added and the reaction was stirred for $20 \mathrm{~min}$. Benzyl bromide $(1.30 \mathrm{~mL}, 10.76 \mathrm{mmol})$ was added and the reaction was slowly warmed to room temperature overnight. After $15 \mathrm{~h}$, the reaction was quenched with water and extracted with ether $(4 \times 30 \mathrm{~mL})$. The combined organic layers were dried with $\mathrm{MgSO}_{4}$, filtered and concentrated. The resulting oil was purified by flash chromatography $(15 \%$ ether in pentane, $\mathrm{R}_{\mathrm{f}}=0.45$ in $40 \%$ EtOAc in Hex) yielding $637.2 \mathrm{mg}(63 \%)$ of $\mathbf{3}$ as a colorless oil. ${ }^{1} \mathrm{H}$ NMR $\left(400 \mathrm{MHz}, \mathrm{CDCl}_{3}\right) \delta 2.59-2.65(\mathrm{dt}, \mathrm{J}=6.4 \mathrm{~Hz}, 12.7 \mathrm{~Hz}, 2 \mathrm{H}), 3.69(\mathrm{q}, \mathrm{J}=6.4$ $\mathrm{Hz}, 2 \mathrm{H}), 4.58(\mathrm{~s}, 2 \mathrm{H}), 7.28-7.39(\mathrm{~m}, 5 \mathrm{H}) .{ }^{13} \mathrm{C} \mathrm{NMR}\left(125.6 \mathrm{MHz}, \mathrm{CDCl}_{3}\right) \delta 19.1(\mathrm{~d}, \mathrm{~J}=$ $\left.57.9 \mathrm{~Hz}, \mathrm{CH}_{2}\right), 64.8\left(\mathrm{~d}, \mathrm{~J}=2.8 \mathrm{~Hz}, \mathrm{CH}_{2}\right), 73.5\left(\mathrm{~s}, \mathrm{CH}_{2}\right), 118.0$ (s, Cq, Enhanced Signal), $128.0(\mathrm{~s}, \mathrm{CH}), 128.3(\mathrm{~s}, \mathrm{CH}), 128.8(\mathrm{~s}, \mathrm{CH}), 137.4$ (d, J = 1.7 Hz, Cq). HRMS (EI, M) for ${ }^{13} \mathrm{CC}_{9} \mathrm{H}_{11} \mathrm{NO}$ calcd 162.0874 , found 162.0872 .

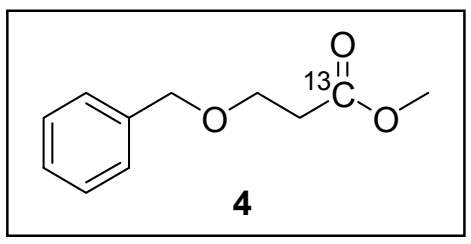

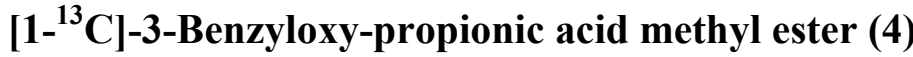

A flask was charged with $3(407.1 \mathrm{mg}, 2.52 \mathrm{mmol})$. Methanol $(10 \mathrm{~mL})$ was added and the solution was cooled to $0{ }^{\circ} \mathrm{C}$. $\mathrm{HCl}(\mathrm{g})$ was bubbled through for $10 \mathrm{~min}$, and the 
reaction was stirred for an additional $60 \mathrm{~min}$, and then stored overnight without stirring in a $4{ }^{\circ} \mathrm{C}$ refrigerator. The methanol was removed under reduced pressure and $\mathrm{CH}_{2} \mathrm{Cl}_{2}(15$ $\mathrm{mL}$ ) was added. The solution was cooled to $0{ }^{\circ} \mathrm{C}$ and $1 \mathrm{M} \mathrm{HCl}(5 \mathrm{~mL}$, aq) was added. The reaction was stirred for $4 \mathrm{~h}$ and then extracted with $\mathrm{CH}_{2} \mathrm{Cl}_{2}(4 \times 30 \mathrm{~mL})$. The combined organic layers were dried $\mathrm{MgSO}_{4}$, filtered and concentrated under vacuum. The oil was purified by flash chromatography $\left(30 \% \mathrm{Et}_{2} \mathrm{O}\right.$ in pentane, $\mathrm{R}_{\mathrm{f}}=0.35$ in $40 \%$ ether in Hex), yielding $387.6 \mathrm{mg}(79 \%)$ of $\mathbf{4}$ as a clear oil. ${ }^{1} \mathrm{H}$ NMR $\left(400 \mathrm{MHz}, \mathrm{CDCl}_{3}\right) \delta$ $2.63(\mathrm{q}, \mathrm{J}=6.5 \mathrm{~Hz}, 2 \mathrm{H}), 3.70(\mathrm{~d}, \mathrm{~J}=3.9 \mathrm{~Hz}, 3 \mathrm{H}), 3.72-3.77(\mathrm{~m}, 2 \mathrm{H}), 4.54(\mathrm{~s}, 2 \mathrm{H}), 7.27-$ $7.37(\mathrm{~m}, 5 \mathrm{H}) .{ }^{13} \mathrm{C}$ NMR $\left(100.6 \mathrm{MHz}, \mathrm{CDCl}_{3}\right) \delta 35.2\left(\mathrm{~d}, \mathrm{~J}=57.9 \mathrm{~Hz}, \mathrm{CH}_{2}\right), 51.9(\mathrm{~d}, \mathrm{~J}=$ $\left.3.1 \mathrm{~Hz}, \mathrm{CH}_{3}\right), 65.8\left(\mathrm{~d}, \mathrm{~J}=1.6 \mathrm{~Hz}, \mathrm{CH}_{2}\right), 73.3\left(\mathrm{~s}, \mathrm{CH}_{2}\right), 127.90(\mathrm{~s}, \mathrm{CH}), 127.92(\mathrm{~s}, \mathrm{CH})$, 128.6 (s, CH), 138.2 (s, Cq), 172.3 (s, Cq, Enhanced Signal). HRMS (EI, M) for ${ }^{13} \mathrm{CC}_{10} \mathrm{H}_{14} \mathrm{O}_{3}$ calcd 195.0976, found 195.0973.

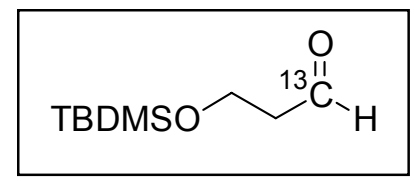

\section{$\left[1-{ }^{13} \mathrm{C}\right]-3$-(tert-Butyl-dimethyl-silanyloxy)-propionaldehyde (2b)}

To a flask charged with $4(408.3 \mathrm{mg}, 2.10 \mathrm{mmol})$ was added $10 \mathrm{~mL}$ of methanol and $\mathrm{Pd} / \mathrm{C}(\sim 5 \mathrm{mg})$. The suspension was stirred under a $\mathrm{H}_{2}$ atmosphere of $150 \mathrm{psi}$ for $8 \mathrm{~h}$ in a reinforced reaction vessel. The vessel was vented and the solution filtered, washed with ether and concentrated. The resulting clear oil was taken on without further purification. ${ }^{1} \mathrm{H}$ NMR $\left(500 \mathrm{MHz}, \mathrm{CDCl}_{3}\right) \delta$ 2.53-2.58 (m, 2H), $3.69(\mathrm{~d}, \mathrm{~J}=3.9 \mathrm{~Hz}, 3 \mathrm{H}), 3.84(\mathrm{q}, \mathrm{J}=$ $5.9 \mathrm{~Hz}, 2 \mathrm{H}) .{ }^{13} \mathrm{C} \mathrm{NMR}\left(125.7 \mathrm{MHz}, \mathrm{CDCl}_{3}\right) \delta 36.9\left(\mathrm{~d}, \mathrm{~J}=56.9 \mathrm{~Hz}, \mathrm{CH}_{2}\right), 51.8\left(\mathrm{~m}, \mathrm{CH}_{3}\right)$, $58.3\left(\mathrm{~d}, \mathrm{~J}=2.8 \mathrm{~Hz}, \mathrm{CH}_{2}\right.$ ), 173.4 (s, Cq, Enhanced Signal).

A flask charged with the 3-hydroxy-propionic acid methyl ester was purged with argon. $\mathrm{CH}_{2} \mathrm{Cl}_{2}(15 \mathrm{~mL})$ was added, and the solution was cooled to $0{ }^{\circ} \mathrm{C}$. Imidazole $(464.3 \mathrm{mg}$, $6.80 \mathrm{mmol}$ ) was added followed by tert-butyldimethylsilyl chloride $(1.04 \mathrm{~g}, 6.90 \mathrm{mmol})$. The reaction was stirred at $0{ }^{\circ} \mathrm{C}$ for $3 \mathrm{~h}$ and then slowly warmed to room temperature overnight. Water $(20 \mathrm{~mL})$ was added and the mixture was extracted with $\mathrm{CH}_{2} \mathrm{Cl}_{2}(3 \times 40$ $\mathrm{mL}$ ). The organic layers were dried with $\mathrm{MgSO}_{4}$, filtered, and concentrated under vacuum. The oil was purified by flash chromatography $\left(5 \% \mathrm{Et}_{2} \mathrm{O}\right.$ in pentane, $\mathrm{R}_{\mathrm{f}}=0.44$ in $10 \%$ EtOAc in Hex) yielding $381.9 \mathrm{mg}$ (83\% over 2 steps) of 3-(tert-butyl-dimethylsilanyloxy)-propionic acid methyl ester as a clear oil. ${ }^{1} \mathrm{H}$ NMR $\left(400 \mathrm{MHz}, \mathrm{CDCl}_{3}\right) \delta 0.04$ $(\mathrm{s}, 6 \mathrm{H}), 0.86(\mathrm{~s}, 9 \mathrm{H}), 2.51(\mathrm{q}, \mathrm{J}=6.6 \mathrm{~Hz}, 2 \mathrm{H}), 3.66(\mathrm{~d}, \mathrm{~J}=3.9 \mathrm{~Hz}, 3 \mathrm{H}), 3.85-3.91(\mathrm{~m}$, $2 \mathrm{H}) .{ }^{13} \mathrm{C}$ NMR $\left(100.6 \mathrm{MHz}, \mathrm{CDCl}_{3}\right) \delta-5.3\left(\mathrm{~s}, \mathrm{CH}_{3}\right), 26.0\left(\mathrm{~s}, \mathrm{CH}_{3}\right), 38.1(\mathrm{~d}, \mathrm{~J}=57.5 \mathrm{~Hz}$, $\left.\mathrm{CH}_{2}\right), 51.7\left(\mathrm{~d}, \mathrm{~J}=2.3 \mathrm{~Hz}, \mathrm{CH}_{3}\right), 59.3\left(\mathrm{~d}, \mathrm{~J}=2.2 \mathrm{~Hz}, \mathrm{CH}_{2}\right), 172.4(\mathrm{~s}, \mathrm{Cq}$, Enhanced Signal).

A flask charged with 3-(tert-butyl-dimethyl-silanyloxy)-propionic acid methyl ester (285.6 mg, $1.29 \mathrm{mmol}$ ) was purged with argon. $\mathrm{CH}_{2} \mathrm{Cl}_{2}(10 \mathrm{~mL})$ was added, and the solution was cooled to $-78{ }^{\circ} \mathrm{C}$. A $1.0 \mathrm{M}$ solution of DIBAL in $\mathrm{CH}_{2} \mathrm{Cl}_{2}(1.42 \mathrm{~mL}, 1.42$ mmol) was added dropwise and the reaction was stirred for $1.5 \mathrm{~h}$. The reaction was quenched with $0.5 \mathrm{~mL}$ of methanol and warmed to room temperature. Rochelle's salt (10 $\mathrm{mL}$, sat. aqueous) and ether $(20 \mathrm{~mL})$ were added and the biphasic solution stirred for 45 min. The solution was extracted with ether $(3 \times 30 \mathrm{~mL})$, and the combined organic layers 
were dried with $\mathrm{MgSO}_{4}$, filtered, and concentrated under vacuum. The resulting oil was purified by flash chromatography $\left(15 \%\right.$ ether in pentane, $\mathrm{R}_{\mathrm{f}}=0.63$ in $20 \%$ EtOAc in Hex) yielding $240.4 \mathrm{mg}(98 \%)$ of $\mathbf{2 b}$ as a colorless oil. ${ }^{1} \mathrm{H}$ NMR $\left(400 \mathrm{MHz}, \mathrm{CDCl}_{3}\right) \delta$ $0.06(\mathrm{~s}, 6 \mathrm{H}), 0.88$ (s, 9H), 2.60 (dq, J = 2.0 Hz, 6.1 Hz, 2H), 3.99 (q, J = 6.0 Hz, 2H), 9.80 $(\mathrm{td}, \mathrm{J}=2.0 \mathrm{~Hz}, 172.6 \mathrm{~Hz}, 1 \mathrm{H}) .{ }^{13} \mathrm{C} \mathrm{NMR}\left(100.6 \mathrm{MHz}, \mathrm{CDCl}_{3}\right) \delta-5.2(\mathrm{~s}, \mathrm{CH} 3), 18.4(\mathrm{~s}$, $\mathrm{Cq}), 26.0\left(\mathrm{~s}, \mathrm{CH}_{3}\right), 46.8$ (d, J = 39.7 Hz, $\left.\mathrm{CH}_{2}\right), 57.6$ (d, J = 1.6 Hz, $\left.\mathrm{CH}_{2}\right), 202.3$ (s, CH, Enhanced Signal). IR: 2931, 2860, 1693, 1472. HRMS (CI, M+1) for ${ }^{13} \mathrm{CC}_{8} \mathrm{H}_{21} \mathrm{O}_{2} \mathrm{Si}$ calcd 190.1344, found 190.1341.

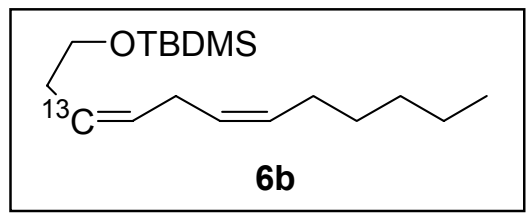

\section{[3- $\left.{ }^{13} \mathrm{C}\right]$-tert-Butyl-dodeca-3,6-dienyloxy-dimethyl-silane (6b)}

A flask charged with phosphonium salt $5(355.0 \mathrm{mg}, 0.70 \mathrm{mmol})$ was purged with argon. THF $(8 \mathrm{~mL})$ was added and the solution was cooled to $0{ }^{\circ} \mathrm{C}$. A solution of $1.0 \mathrm{M}$ NaHMDS in THF $(700 \mu \mathrm{L}, 0.63 \mathrm{mmol})$ was added, and the reaction was stirred for 30 min and then cooled to $-78{ }^{\circ} \mathrm{C}$. Compound $2 \mathbf{b}(66.5 \mathrm{mg}, 0.35 \mathrm{mmol})$ in $3 \mathrm{~mL}$ of $\mathrm{THF}$ was added dropwise and the reaction was stirred at $-78^{\circ} \mathrm{C}$ for $6 \mathrm{~h}$, then slowly warmed to room temperature overnight. $\mathrm{H}_{2} \mathrm{O}(10 \mathrm{~mL})$ was added and the solution was extracted with ether $(4 \times 30 \mathrm{~mL})$. The combined organic layers were dried with $\mathrm{MgSO}_{4}$, filtered and concentrated. The residual oil was purified by flash chromatography ( $100 \%$ pentane, $\mathrm{R}_{\mathrm{f}}=0.37$ in $100 \%$ pentane) yielding $95.5 \mathrm{mg}(91 \%)$ of $\mathbf{6 b}$ as a clear oil. ${ }^{1} \mathrm{H}$ NMR (500 $\left.\mathrm{MHz}, \mathrm{CDCl}_{3}\right) \delta 0.06(\mathrm{~s}, 6 \mathrm{H}), 0.90(\mathrm{~s}, 9 \mathrm{H}), 1.23-1.39(\mathrm{~m}, 6 \mathrm{H}), 2.05(\mathrm{q}, \mathrm{J}=7.0 \mathrm{MHz}, 2 \mathrm{H})$, 2.30 (pentet, $\mathrm{J}=7.0 \mathrm{MHz}, 2 \mathrm{H}), 2.79(\mathrm{q}, \mathrm{J}=6.4 \mathrm{MHz}, 2 \mathrm{H}), 3.62(\mathrm{dt}, \mathrm{J}=3.8 \mathrm{MHz}, 7.1$ $\mathrm{MHz}, 2 \mathrm{H}), 5.20-5.26(\mathrm{~m}, 0.5 \mathrm{H}), 5.30-5.45(\mathrm{~m}, 3 \mathrm{H}), 5.52-5.57(\mathrm{~m}, 0.5 \mathrm{H}) .{ }^{13} \mathrm{C} \mathrm{NMR}$ $\left(125.6 \mathrm{MHz}, \mathrm{CDCl}_{3}\right) \delta-5.1\left(\mathrm{~s}, \mathrm{CH}_{3}\right), 14.3\left(\mathrm{~s}, \mathrm{CH}_{3}\right), 18.6(\mathrm{~s}, \mathrm{Cq}), 22.8\left(\mathrm{~s}, \mathrm{CH}_{2}\right), 26.0(\mathrm{~s}$, $\mathrm{Cq}), 26.2\left(\mathrm{~s}, \mathrm{CH}_{3}\right), 27.4\left(\mathrm{~s}, \mathrm{CH}_{2}\right), 29.5\left(\mathrm{~s}, \mathrm{CH}_{2}\right), 31.3\left(\mathrm{~d}, \mathrm{~J}=42.3 \mathrm{MHz}, \mathrm{CH}_{2}\right), 31.7(\mathrm{~s}$, $\mathrm{CH}_{2}$ ), 63.1 (s, $\mathrm{CH}_{2}$ ), 126.1 (s, CH, Enhanced Signal). 126.22, 126.29, 126.3, 127.91, $127.94,129.9,130.5,130.6$. No efforts were made to assign peaks in the vinyl region which show splitting patterns as a result of the presence of the ${ }^{13} \mathrm{C}$ nucleus: 126.22 , 126.29, 126.3, 127.91, 127.94, 129.9, 130.5, and 130.6 ppm. IR: 2958, 2930, 2859, 1472, 1256. HRMS (CI, M+1) for ${ }^{13} \mathrm{CC}_{17} \mathrm{H}_{37} \mathrm{OSi}$ calcd 298.2647, found 298.2645.

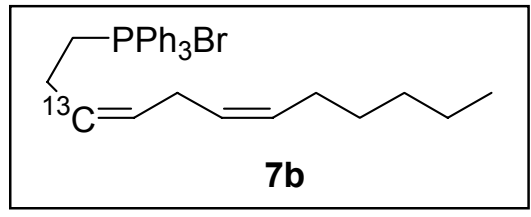

\section{Phosphonium salt of $\left[3-{ }^{13} \mathrm{C}\right]-1-b r o m o-d o d e c a-3,6-d i e n e ~(7 b)$}

A flask charged with silyl protected alcohol $\mathbf{6 b}$ was purged with argon. $\mathrm{CH}_{2} \mathrm{Cl}_{2}(2 \mathrm{~mL})$ was added followed by the addition of a $1.0 \mathrm{M}$ solution of TBAF in THF $(0.50 \mathrm{~mL}, 0.40$ $\mathrm{mmol})$. The reaction was stirred for $15 \mathrm{~h}$ then the solvent was removed under vacuum. The resulting oil was purified by flash chromatography $\left(20 \%\right.$ ether in pentane, $\mathrm{R}_{\mathrm{f}}=0.43$ in $20 \%$ EtOAc in Hex ) yielding $50.9 \mathrm{mg}(97 \%)$ of dodeca-3,6-dien-1-ol as a clear oil. 
${ }^{1} \mathrm{H}$ NMR (400 MHz, $\left.\mathrm{CDCl}_{3}\right) \delta 0.89(\mathrm{t}, \mathrm{J}=6.9 \mathrm{~Hz}, 3 \mathrm{H}), 1.24-1.40(\mathrm{~m}, 6 \mathrm{H}), 2.05(\mathrm{q}, \mathrm{J}=$ $7.0 \mathrm{~Hz}, 2 \mathrm{H}), 2.36$ (pentet, $\mathrm{J}=6.4 \mathrm{~Hz}, 2 \mathrm{H}), 2.82(\mathrm{q}, \mathrm{J}=6.3 \mathrm{~Hz}, 2 \mathrm{H}), 3.66(\mathrm{dt}, \mathrm{J}=4.6 \mathrm{~Hz}$, $6.4 \mathrm{~Hz}, 2 \mathrm{H}), 5.16-5.24(\mathrm{~m}, 0.5 \mathrm{H}), 5.28-5.44(\mathrm{~m}, 2 \mathrm{H}), 5.50-5.62(\mathrm{~m}, 0.5 \mathrm{H}) .{ }^{13} \mathrm{C} \mathrm{NMR}$ $\left(125.6 \mathrm{MHz}, \mathrm{CDCl}_{3}\right) \delta 14.3\left(\mathrm{~s}, \mathrm{CH}_{3}\right), 22.8\left(\mathrm{~s}, \mathrm{CH}_{2}\right), 25.9\left(\mathrm{~s}, \mathrm{CH}_{2}\right), 27.4\left(\mathrm{~s}, \mathrm{CH}_{2}\right), 29.5(\mathrm{~s}$, $\mathrm{CH}_{2}$ ), $31.0\left(\mathrm{~d}, \mathrm{~J}=41.0 \mathrm{~Hz}, \mathrm{CH}_{2}\right), 31.7\left(\mathrm{~s}, \mathrm{CH}_{2}\right), 62.5$ (d, J = 2.8 Hz, $\left.\mathrm{CH}_{2}\right), 125.2,125.3$, 125.5 (s, CH, Enhanced Signal), 125.8, 126.3, 127.56, 127.60, 130.9, 132.1. No efforts were made to assign peaks in the vinyl region which show splitting patterns as a result of the presence of the ${ }^{13} \mathrm{C}$ nucleus. IR: 3369 (br), 3010, 2929, $1468 \mathrm{~cm}^{-1}$. HRMS (EI) calcd for ${ }^{13} \mathrm{CC}_{11} \mathrm{H}_{22} \mathrm{O} 183.1704$, found 183.1699 .

A flask charged with triphenylphosphine $(88.2 \mathrm{mg}, 0.32 \mathrm{mmol})$ was purged with argon. $\mathrm{CH}_{2} \mathrm{Cl}_{2}(6 \mathrm{~mL})$ was added and the solution cooled to $-10{ }^{\circ} \mathrm{C}$. Bromine $(17 \mu \mathrm{L}, 0.32$ mmol) was added, and the reaction was stirred for $20 \mathrm{~min}$. Following addition of pyridine $(26 \mu \mathrm{L}, 0.32 \mathrm{mmol})$ the reaction was stirred an additional $20 \mathrm{~min}$ then the dodeca-3,6-dien-1-ol (45.5 mg, $0.25 \mathrm{mmol}$ ) in $1 \mathrm{~mL}$ of $\mathrm{CH}_{2} \mathrm{Cl}_{2}$ was added dropwise. After $2 \mathrm{~h}$, the reaction was quenched by the addition of water and extracted with ether ( 3 x $40 \mathrm{~mL}$ ). The combined organic layers were dried with $\mathrm{MgSO}_{4}$, filtered and concentrated. The resulting oil was purified by flash chromatography $\left(100 \%\right.$ pentane, $\mathrm{R}_{\mathrm{f}}$ $=0.53$ in $100 \%$ pentane) yielding $51.7 \mathrm{mg}(85 \%)$ of 1-bromo-dodeca-3,6-diene as a clear oil. ${ }^{1} \mathrm{H}$ NMR $\left(400 \mathrm{MHz}, \mathrm{CDCl}_{3}\right), \delta 0.89(\mathrm{t}, \mathrm{J}=7.0 \mathrm{~Hz}, 3 \mathrm{H}), 1.24-1.40(\mathrm{~m}, 6 \mathrm{H}), 2.05(\mathrm{q}, \mathrm{J}$ $=7.1 \mathrm{~Hz}, 2 \mathrm{H}), 2.65-2.69(\mathrm{~m}, 2 \mathrm{H}), 2.79(\mathrm{q}, \mathrm{J}=6.6 \mathrm{~Hz}, 2 \mathrm{H}), 3.37(\mathrm{dt}, \mathrm{J}=4.1 \mathrm{~Hz}, 7.1 \mathrm{~Hz}$, $2 \mathrm{H}), 5.16-5.23(\mathrm{~m}, 0.50 \mathrm{H}), 5.28-5.45(\mathrm{~m}, 2 \mathrm{H}), 5.48-5.62(\mathrm{~m}, 1.5 \mathrm{H}) .{ }^{13} \mathrm{C} \mathrm{NMR}(100.6$ $\left.\mathrm{MHz}, \mathrm{CDCl}_{3}\right) \delta 14.3\left(\mathrm{~s}, \mathrm{CH}_{3}\right), 22.8\left(\mathrm{~s}, \mathrm{CH}_{2}\right), 26.0\left(\mathrm{~s}, \mathrm{CH}_{2}\right), 27.5\left(\mathrm{~s}, \mathrm{CH}_{2}\right), 29.5\left(\mathrm{~s}, \mathrm{CH}_{2}\right)$, $31.0\left(\mathrm{~d}, \mathrm{~J}=42.5 \mathrm{~Hz}, \mathrm{CH}_{2}\right), 31.7\left(\mathrm{~s}, \mathrm{CH}_{2}\right), 32.6\left(\mathrm{~d}, \mathrm{~J}=1.6 \mathrm{~Hz}, \mathrm{CH}_{2}\right), 125.9,126.3(\mathrm{~s}, \mathrm{CH}$, Enhanced Signal), 126.5, 126.6, 127.0, 127.25, 127.30. No efforts were made to assign peaks in the vinyl region which are split as a result of the presence of the ${ }^{13} \mathrm{C}$ nucleus. IR: 3010, 2959, 2928, 2857, $1456 \mathrm{~cm}^{-1}$. HRMS (CI, M+) for ${ }^{13} \mathrm{CC}_{11} \mathrm{H}_{21} \mathrm{Br}$ calcd 245.0860, found 245.0858 .

A flask charged with 1-bromo-dodeca-3,6-diene was flushed with argon. Acetonitrile (2 $\mathrm{mL})$ was added followed by triphenylphosphine $(85.2 \mathrm{mg}, 0.34 \mathrm{mmol})$. The reaction was heated at $90{ }^{\circ} \mathrm{C}$ for $19 \mathrm{~h}$. After cooling to room temperature, the reaction was poured onto a column impregnated with $1 / 1$ pentane/ether. After elution of excess triphenylphosphine, the product was eluted using 5\% methanol in $\mathrm{CH}_{2} \mathrm{Cl}_{2}$ as mobile phase. Yield $61.6 \mathrm{mg}(70 \%)$ of $7 \mathbf{b}$ as a gummy colorless oil. ${ }^{1} \mathrm{H}$ NMR $(500 \mathrm{MHz}$, $\left.\mathrm{CDCl}_{3}\right) \delta 0.81(\mathrm{t}, \mathrm{J}=7.1 \mathrm{~Hz}, 3 \mathrm{H}), 1.11-1.25(\mathrm{~m}, 6 \mathrm{H}), 1.81(\mathrm{q}, \mathrm{J}=7.2 \mathrm{~Hz}, 2 \mathrm{H}), 2.38-2.45$ $(\mathrm{m}, 2 \mathrm{H}), 2.49$ (q, J = 6.8 Hz, 2H), 3.75-3.82 (m, 2H), 5.06-5.13 (m, 1H), 5.24-5.40 (m, 2.5H), 5.67-5.72 (m, 0.5H). ${ }^{13} \mathrm{C}$ NMR $\left(125.6 \mathrm{MHz} \mathrm{CDCl}_{3}\right) \delta 14.1\left(\mathrm{~s}, \mathrm{CH}_{3}\right), 20.6(\mathrm{dd}, \mathrm{J}=$ $\left.3.8 \mathrm{~Hz}, 42.5 \mathrm{~Hz}, \mathrm{CH}_{2}\right), 22.6\left(\mathrm{~s}, \mathrm{CH}_{2}\right), 23.2$ (d, J = 47.6 Hz, $\left.\mathrm{CH}_{2}\right), 25.7\left(\mathrm{~s}, \mathrm{CH}_{2}\right), 27.3(\mathrm{~s}$, $\left.\mathrm{CH}_{2}\right), 29.3\left(\mathrm{~s}, \mathrm{CH}_{2}\right), 31.6\left(\mathrm{~s}, \mathrm{CH}_{2}\right), 118.4(\mathrm{~d}, \mathrm{~J}=85.7 \mathrm{~Hz}, \mathrm{CH}), 126.5(\mathrm{~d}, \mathrm{~J}=14.5 \mathrm{~Hz}, \mathrm{CH}$, Enhanced Signal), 126.7, 126.8, 126.82, 126.85, 126.9, 126.92, 130.6. 130.7 (d, J= 12.9 $\mathrm{Hz}, \mathrm{CH}), 131.0,131.1,133.9$ (d, J = 10.1 Hz, CH), 135.3 (d, J = 2.8 Hz, CH). No efforts were made to assign peaks in the vinyl region which show splitting patterns as a result of the presence of the ${ }^{31} \mathrm{P}$ and ${ }^{13} \mathrm{C}$ nuclei. 


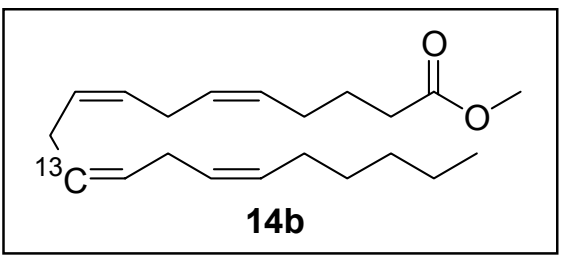

\section{$\left[11-{ }^{13} \mathrm{C}\right]-$ Methyl arachidonate (14b)}

Triphenylphosphonium salt $7 \mathbf{b}(40.0 \mathrm{mg}, 0.078 \mathrm{mmol})$ was dried four times by azeotropic evaporation with anhydrous benzene. THF $(2 \mathrm{~mL})$ was added and the solution was cooled to $-78^{\circ} \mathrm{C}$. A solution of $1.0 \mathrm{M}$ NaHMDS $(74 \mu \mathrm{L}, 0.074 \mathrm{mmol})$ was added and the reaction was stirred for $60 \mathrm{~min}$. The solution was cooled to $-90{ }^{\circ} \mathrm{C}$, and aldehyde $\mathbf{1 3}$ (20.3 mg, $0.122 \mathrm{mmol}$ ) in $1 \mathrm{~mL}$ of THF was added dropwise. The reaction was stirred at $-78{ }^{\circ} \mathrm{C}$ for $5 \mathrm{~h}$ then slowly warmed to room temperature overnight. The reaction was quenched with water and extracted with ether $(4 \times 20 \mathrm{~mL})$. The combined organic layers were washed with water $(1 \times 5 \mathrm{~mL})$, dried with $\mathrm{MgSO}_{4}$, filtered and concentrated. The resulting oil was purified by flash chromatography (5\% ether in pentane, $\mathrm{R}_{\mathrm{f}}=0.50$ in $5 \%$ EtOAc in Hex) yielding $17.0 \mathrm{mg}(71 \%)$ of $\mathbf{1 4 b}$ as a clear oil. ${ }^{1} \mathrm{H}$ NMR $(500 \mathrm{MHz}$, $\left.\mathrm{CDCl}_{3}\right) \delta 0.89(\mathrm{t}, \mathrm{J}=7.0 \mathrm{~Hz}, 3 \mathrm{H}), 1.24-1.40(\mathrm{~m}, 8 \mathrm{H}), 1.71$ (quintet, $\left.\mathrm{J}=7.4 \mathrm{~Hz}, 2 \mathrm{H}\right), 2.05$ (quartet, $\mathrm{J}=7.1 \mathrm{~Hz}, 2 \mathrm{H}$ ), 2.11 (quartet, $\mathrm{J}=7.1 \mathrm{~Hz}, 2 \mathrm{H}$ ), 2.32 (t, J = 7.5 Hz, 2H), 2.78 $2.86(\mathrm{~m}, 6 \mathrm{H}), 3.67(\mathrm{~s}, 3 \mathrm{H}), 5.18-5.24(\mathrm{~m}, 0.5 \mathrm{H}), 5.30-5.44(\mathrm{~m}, 7 \mathrm{H}), 5.48-5.54(\mathrm{~m}, 0.5 \mathrm{H})$. ${ }^{13} \mathrm{C} \mathrm{NMR}\left(125.6 \mathrm{MHz}, \mathrm{CDCl}_{3}\right) \delta 14.3\left(\mathrm{CH}_{3}\right), 22.8\left(\mathrm{CH}_{2}\right), 25.0\left(\mathrm{CH}_{2}\right), 25.8(\mathrm{~d}, \mathrm{~J}=42.3$ $\left.\mathrm{Hz}, \mathrm{CH}_{2}\right), 25.81\left(\mathrm{~d}, \mathrm{~J}=3.0 \mathrm{~Hz}, \mathrm{CH}_{2}\right), 26.7\left(\mathrm{CH}_{2}\right), 27.4\left(\mathrm{CH}_{2}\right), 29.5\left(\mathrm{CH}_{2}\right), 30.5\left(\mathrm{CH}_{2}\right)$, $31.7\left(\mathrm{CH}_{2}\right), 33.6\left(\mathrm{CH}_{2}\right), 51.7\left(\mathrm{CH}_{3}\right), 125.7,127.8,127.9,127.92,127.95,128.08(\mathrm{CH}$, Enhanced Signal), 128.3, 128.4, 128.6, 129.1, 129.2, 130.7, 131.9, 174.3 (Cq).

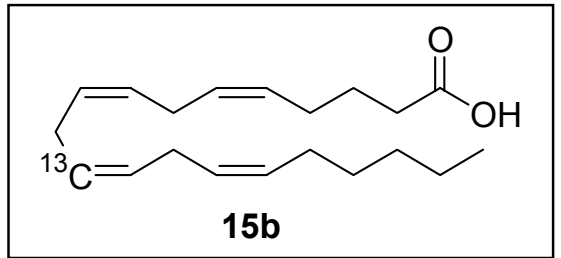

\section{$\left[11-{ }^{13} \mathrm{C}\right]$-Aracidonic Acid (15b)}

A flask was charged with $\mathbf{1 4 b}(5.2 \mathrm{mg}, 0.017 \mathrm{mmol})$ and flushed with argon. To this THF $(1 \mathrm{~mL})$ was added and the mixture was cooled to $0{ }^{\circ} \mathrm{C}$. A solution of $1.0 \mathrm{M}$ aqueous $\mathrm{LiOH}(0.25 \mathrm{~mL})$ was added dropwise and the reaction was stirred for $1 \mathrm{~h}$, then slowly warmed to room temperature. After $16 \mathrm{~h}$, the $\mathrm{pH}$ of the reaction was adjusted to $\mathrm{pH}=1$ using $1 \mathrm{M} \mathrm{HCl}$ and the solution was extracted with ether $(3 \times 10 \mathrm{~mL})$. The combined organic layers were washed with water $(1 \times 5 \mathrm{~mL})$, dried with $\mathrm{MgSO}_{4}$, filtered and concentrated. The resulting oil was purified by HPLC, using the conditions previously described for purification of $\left[10,10-{ }^{2} \mathrm{H}_{2}\right]$-arachidonic acid yielding $4.5 \mathrm{mg}(90 \%)$ of $\mathbf{1 5 b}$ as a clear oil. ${ }^{1} \mathrm{H}$ NMR $\left(500 \mathrm{MHz}, \mathrm{CDCl}_{3}\right) \delta 0.89(\mathrm{t}, \mathrm{J}=7.0 \mathrm{~Hz}, 3 \mathrm{H}), 1.26-1.40(\mathrm{~m}, 8 \mathrm{H})$, 1.73 (pentet, $\mathrm{J}=7.4 \mathrm{~Hz}, 2 \mathrm{H}$ ), 2.06 (quartet, $\mathrm{J}=7.2 \mathrm{~Hz}, 2 \mathrm{H}$ ), 2.15 (quartet, $\mathrm{J}=7.1 \mathrm{~Hz}$, $2 \mathrm{H}), 2.38(\mathrm{t}, \mathrm{J}=7.6 \mathrm{~Hz}, 2 \mathrm{H}), 2.80-2.87(\mathrm{~m}, 6 \mathrm{H}), 5.18-5.24(\mathrm{~m}, 0.5 \mathrm{H}), 5.31-5.45(\mathrm{~m}, 7 \mathrm{H})$, 5.49-5.55 (m, 0.5H). ${ }^{13} \mathrm{C}$ NMR $\left(125.6 \mathrm{MHz} \mathrm{CDCl}_{3}\right) \delta 128.1$ (s, Enhanced Signal, $\left.\mathrm{CH}\right)$. 


\section{$\left[15{ }^{13} \mathrm{C}\right]$-Arachidonic Acid}

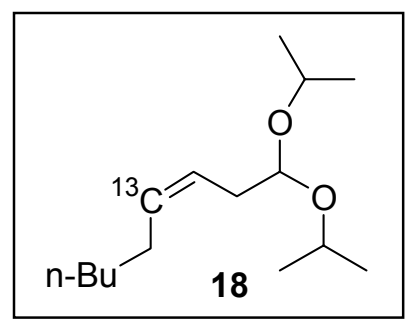

\section{4- ${ }^{13} \mathrm{C}-1,1-D i i s o p r o p o x y-n o n-3-e n e ~(18)$}

(3,3-Diisopropoxy-propyl)-triphenyl-phosphonium bromide 16 (536 $\mathrm{mg}, 1.1 \mathrm{mmol}$ ) was dried three times by azeotropic distillation under reduced pressure with anhydrous dry benzene. After purging with Ar, THF $(15 \mathrm{~mL})$ was added, followed by a $1 \mathrm{M}$ solution of sodium bis(trimethylsilyl)amide $(0.96 \mathrm{~mL}, 0.96 \mathrm{mmol})$ in THF at $-40{ }^{\circ} \mathrm{C}$. The orange ylide was stirred at $-40{ }^{\circ} \mathrm{C}$ for $10 \mathrm{~min}$, then continued to stir at room temperature for $1 \mathrm{~h}$. The mixture was cooled to $-100{ }^{\circ} \mathrm{C}$ and $1-{ }^{13} \mathrm{C}$-hexanal 17 (90 mg, $\left.0.9 \mathrm{mmol}\right)$ in THF (2 $\mathrm{mL}$ ) was added slowly via syringe. The reaction was allowed to warm to $0{ }^{\circ} \mathrm{C}$ over $4 \mathrm{~h}$. Saturated ammonium chloride solution was added to quench the reaction. After extraction with $\mathrm{Et}_{2} \mathrm{O}(3 \times 50 \mathrm{~mL})$, the combined organic layers were dried with $\mathrm{NaSO}_{4}$, concentrated under reduced pressure, and the crude product was purified by flash chromatography $\left(\mathrm{R}_{\mathrm{f}}\right.$ 0.5, hexane:EtOAc $=100: 5)$ to provide $4-{ }^{13} \mathrm{C}$-1,1-diisopropoxy-non-3-ene $\mathbf{1 8}(130 \mathrm{mg}$, $60 \%)$ as a colorless oil. ${ }^{1} \mathrm{H}$ NMR $\left(500 \mathrm{MHz}, \mathrm{CDCl}_{3}\right) \delta 5.66-5.61(0.5 \mathrm{H}, \mathrm{m}), 5.43-5.30$ $(1.5 \mathrm{H}, \mathrm{m}), 4.53(1 \mathrm{H}, \mathrm{t}, \mathrm{J}=6.0 \mathrm{~Hz}), 3.88(1 \mathrm{H}$, sept, J=6.3 Hz), 2.38-2.34 $(2 \mathrm{H}, \mathrm{m}), 2 . .04$ $(2 \mathrm{H}$, quin, J=7.9 Hz), $1.37(6 \mathrm{H}, \mathrm{d}, \mathrm{J}=6.3 \mathrm{~Hz}), 1.21(6 \mathrm{H}, \mathrm{d}, \mathrm{J}=6.2 \mathrm{~Hz}), 0.90(3 \mathrm{H}, \mathrm{t}, \mathrm{J}=7.0$ $\mathrm{Hz}) .{ }^{13} \mathrm{C}$ NMR $\left(125.6 \mathrm{MHz}, \mathrm{CDCl}_{3}\right) \delta 14.3\left(\mathrm{~s}, \mathrm{CH}_{3}\right), 22.7\left(\mathrm{~s}, \mathrm{CH}_{3}\right), 22.8\left(\mathrm{~s}, \mathrm{CH}_{3}\right), 23.6(\mathrm{~s}$, $\left.\mathrm{CH}_{2}\right), 27.7\left(\mathrm{~d}, \mathrm{~J}=42.3 \mathrm{~Hz}, \mathrm{CH}_{2}\right), 29.5\left(\mathrm{~d}, \mathrm{~J}=1.8 \mathrm{~Hz}, \mathrm{CH}_{2}\right), 31.7\left(\mathrm{~d}, \mathrm{~J}=3.6 \mathrm{~Hz}, \mathrm{CH}_{2}\right)$, $33.9\left(\mathrm{~s}, \mathrm{CH}_{2}\right), 68.0(\mathrm{~s}, \mathrm{CH}), 100.3(\mathrm{~s}, \mathrm{CH}), 124.4(\mathrm{~d}, \mathrm{~J}=70.8 \mathrm{~Hz}, \mathrm{CH}), 132.4(\mathrm{~s}, \mathrm{CH}$, Enhanced Signal).

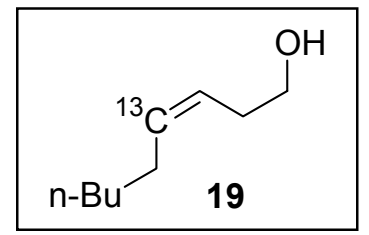

\section{$\left[4-{ }^{13}\right.$ C]-Non-3-en-1-ol (19)}

A mixture of isopropylacetal $18(110 \mathrm{mg}, 0.45 \mathrm{mmol})$ and a $1 \mathrm{M}$ solution of aqueous ptoluenesulfonic acid $(0.22 \mathrm{~mL}, 0.22 \mathrm{mmol})$ in THF $(9 \mathrm{~mL})$ was refluxed for $20 \mathrm{~min}$. The solution was cooled to $0{ }^{\circ} \mathrm{C}$ and hexane $(20 \mathrm{~mL})$ was added, followed by saturated aqueous sodium chloride. The aqueous phase was extracted with hexane $(2 \times 20 \mathrm{~mL})$ and the combined organic phase was washed with saturated aqueous sodium chloride $(2 \times 10$ $\mathrm{mL}$ ), dried over anhydrous $\mathrm{NaSO}_{4}$ and concentrated to give the aldehyde as a colorless oil, which was used directly without further purification.

$\mathrm{NaBH}_{4}(60 \mathrm{mg}, 1.6 \mathrm{mmol})$ dissolved in $\mathrm{MeOH}(2 \mathrm{~mL})$ was added dropwise to a solution of the aldehyde $(64 \mathrm{mg}, 0.45 \mathrm{mmol})$ in $\mathrm{MeOH}(1 \mathrm{~mL})$. The mixture was stirred 
at $0{ }^{\circ} \mathrm{C}$ for $15 \mathrm{~min}$ and $1 \mathrm{M}$ aqueous $\mathrm{HCl}$ was added to adjust the $\mathrm{pH}$ to 3 . The mixture was extracted with $\mathrm{Et}_{2} \mathrm{O}(3 \times 30 \mathrm{~mL})$ and the combined organic layers were washed with saturated $\mathrm{NaHCO}_{3}$, brine, dried over $\mathrm{Na}_{2} \mathrm{SO}_{4}$, and concentrated under reduced pressure. After silica gel flash chromatography $\left(\mathrm{R}_{\mathrm{f}} 0.2\right.$, hexane:EtOAc $\left.=90: 10\right),\left[4-{ }^{13} \mathrm{C}\right]$-non-3-cisen-1-ol $19(41 \mathrm{mg}, 63 \%)$ was obtained as a colorless oil. ${ }^{1} \mathrm{H}$ NMR $\left(500 \mathrm{MHz}, \mathrm{CDCl}_{3}\right) \delta$ 5.75-5.69 (0.5H, m), 5.45-5.31 (1.5H, m), $3.65(2 \mathrm{H}, \mathrm{t}, \mathrm{J}=6.4 \mathrm{~Hz}), 2.37-2.31(2 \mathrm{H}, \mathrm{m}), 2.07$ $(2 \mathrm{H}, \mathrm{q}, \mathrm{J}=7.0 \mathrm{~Hz}), 1.81(1 \mathrm{H}$, br S$), 1.44-1.26(6 \mathrm{H}, \mathrm{m}), 0.90(3 \mathrm{H}, \mathrm{t}, \mathrm{J}=6.9 \mathrm{~Hz}) .{ }^{13} \mathrm{C} \mathrm{NMR}$ $\left(125.6 \mathrm{MHz}, \mathrm{CDCl}_{3}\right) \delta 133.8$ (Enhanced Signal).

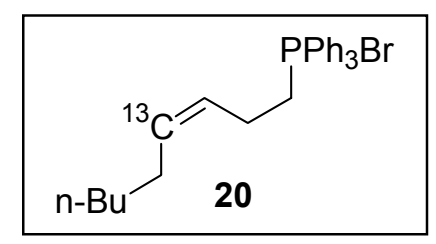

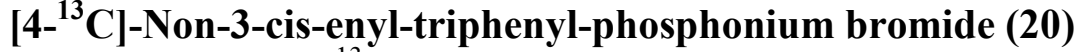

To a solution of [4- ${ }^{13} \mathrm{C}$-non-3-cis-en-1-ol $19(38 \mathrm{mg}, 0.27 \mathrm{mmol})$ and pyridine $(0.06 \mathrm{~mL}$, $0.7 \mathrm{mmol})$ in acetonitrile $(1 \mathrm{~mL})$ was added $\mathrm{PPh}_{3} \mathrm{Br}_{2}(146 \mathrm{mg}, 0.35 \mathrm{mmol})$ at $0{ }^{\circ} \mathrm{C}$. The reaction mixture was stirred at room temperature for $1.5 \mathrm{~h}$ and then filtered through a short plug of silica gel $\left(\mathrm{R}_{\mathrm{f}} 0.6\right.$, hexane) to provide 1 -bromo- $\left[4-{ }^{13} \mathrm{C}\right]$-non-3-cis-ene $(48 \mathrm{mg}$, $88 \%)$ as a colorless oil. ${ }^{1} \mathrm{H}$ NMR $\left(500 \mathrm{MHz}, \mathrm{CDCl}_{3}\right) \delta 5.73-5.67(0.5 \mathrm{H}, \mathrm{m}), 5.43-5.34$ $(1.5 \mathrm{H}, \mathrm{m}), 3.38(2 \mathrm{H}, \mathrm{t}, \mathrm{J}=7.3 \mathrm{~Hz}), 2.66-2.61(2 \mathrm{H}, \mathrm{m}), 2.05(2 \mathrm{H}, \mathrm{q}, \mathrm{J}=7.0 \mathrm{~Hz}), 1.40-1.27$ $(6 \mathrm{H}, \mathrm{m}), 0.91(3 \mathrm{H}, \mathrm{t}, \mathrm{J}=6.9 \mathrm{~Hz}) .{ }^{13} \mathrm{C}$ NMR $\left(125.6 \mathrm{MHz}, \mathrm{CDCl}_{3}\right) \delta 14.3\left(\mathrm{~s}, \mathrm{CH}_{3}\right), 22.8(\mathrm{~s}$, $\left.\mathrm{CH}_{2}\right), 27.6\left(\mathrm{~d}, \mathrm{~J}=42.4 \mathrm{~Hz}, \mathrm{CH}_{2}\right), 29.4\left(\mathrm{~d}, \mathrm{~J}=1.8 \mathrm{~Hz}, \mathrm{CH}_{2}\right), 31.0\left(\mathrm{~s}, \mathrm{CH}_{2}\right), 31.7(\mathrm{~d}, \mathrm{~J}=3.1$ $\left.\mathrm{Hz}, \mathrm{CH}_{2}\right), 32.8\left(\mathrm{~d}, \mathrm{~J}=4.6 \mathrm{~Hz}, \mathrm{CH}_{2}\right), 126.0(\mathrm{~d}, \mathrm{~J}=70.8 \mathrm{~Hz}, \mathrm{CH}), 133.5$ (s, CH, Enhanced Signal).

A mixture of 1-bromo-4-[ $\left.{ }^{13} \mathrm{C}\right]$-non-3-cis-ene $(45 \mathrm{mg}, 0.22 \mathrm{mmol})$ and triphenylphosphine $(116 \mathrm{mg}, 0.44 \mathrm{mmol})$ in acetonitrile $(1 \mathrm{~mL})$ was refluxed for $36 \mathrm{~h}$. The reaction mixture was purified by silica gel flash chromatography $\left(15: 1 \mathrm{CH}_{2} \mathrm{Cl}_{2}\right.$ in methanol) to yield the product $20(86 \mathrm{mg}, 100 \%)$ as a yellow foam. ${ }^{1} \mathrm{H}$ NMR $\left(500 \mathrm{MHz}, \mathrm{CDCl}_{3}\right) \delta$ 7.81-7.65 $(15 \mathrm{H}, \mathrm{m}), 5.50-5.48(1.5 \mathrm{H}, \mathrm{m}), 5.19-5.14(0.5 \mathrm{H}, \mathrm{m}), 3.73-3.72(2 \mathrm{H}, \mathrm{m}), 2.42-2.31(2 \mathrm{H}$, $\mathrm{m}), 1.76(2 \mathrm{H}, \mathrm{q}, \mathrm{J}=7.1 \mathrm{~Hz}), 1.19-1.04(6 \mathrm{H}, \mathrm{m}), 0.77(3 \mathrm{H}, \mathrm{t}, \mathrm{J}=7.2 \mathrm{~Hz}) .{ }^{13} \mathrm{C}$ NMR $(125.6$ $\left.\mathrm{MHz}, \mathrm{CDCl}_{3}\right) \delta 14.2\left(\mathrm{~s}, \mathrm{CH}_{3}\right), 20.5\left(\mathrm{~d}, \mathrm{~J}=3.8 \mathrm{~Hz}, \mathrm{CH}_{2}\right), 22.6\left(\mathrm{~s}, \mathrm{CH}_{2}\right), 23.3(\mathrm{dd}, \mathrm{J}=3.8$ $\left.\mathrm{Hz}, 48.7 \mathrm{~Hz}, \mathrm{CH}_{2}\right), 27.3$ (d, J = 42.6 Hz, $\left.\mathrm{CH}_{2}\right), 29.1\left(\mathrm{~d}, \mathrm{~J}=1.8 \mathrm{~Hz}, \mathrm{CH}_{2}\right), 31.5(\mathrm{~d}, \mathrm{~J}=3.7$ $\left.\mathrm{Hz}, \mathrm{CH}_{2}\right), 118.0,118.6,125.7,125.9,126.3,126.4,128.7,128.8,130.7,130.8$ (d, J = $12.9 \mathrm{~Hz}, \mathrm{CH}), 132.2,132.3,132.5,132.6,132.8$ (s, CH, Enhanced Signal), 133.0, 133.1, $133.9(\mathrm{~d}, \mathrm{~J}=10.1 \mathrm{~Hz}, \mathrm{CH}), 134.2,135.4(\mathrm{~d}, \mathrm{~J}=3.6 \mathrm{~Hz}, \mathrm{CH})$. Due to complex coupling patterns as a result of the presence of both ${ }^{31} \mathrm{P}$ and ${ }^{13} \mathrm{C}$ nuclei, peaks in the aromatic/vinyl region are listed without assignment. 


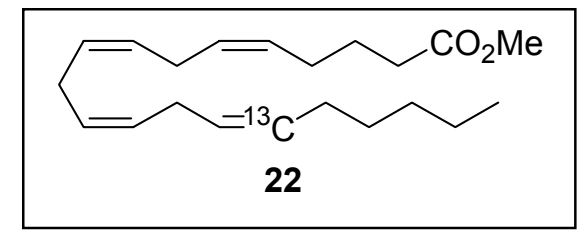

\section{Methyl $\left[15-{ }^{13} \mathrm{C}\right]$-arachidonate (22)}

$\left[4-{ }^{13} \mathrm{C}\right]$-Non-3-cis-enyl-triphenylphosphonium bromide $20(80 \mathrm{mg}, 0.18 \mathrm{mmol})$ was dried three times by azeotropic evaporation with anhydrous benzene. THF $(6 \mathrm{~mL})$ was added, followed by a $1 \mathrm{M}$ solution of sodium bis(trimethylsilyl)amide $(0.16 \mathrm{~mL}, 0.16 \mathrm{mmol})$ in THF at $-40{ }^{\circ} \mathrm{C}$. The orange solution of the ylide was stirred at $-40{ }^{\circ} \mathrm{C}$ for $10 \mathrm{~min}$ and warmed to room temperature over $1 \mathrm{~h}$. The mixture was cooled to $-100{ }^{\circ} \mathrm{C}$, and a solution of 9-oxo-nona-cis,cis-3,6-dienoic acid methyl ester 21 (34 mg, $0.16 \mathrm{mmol})$ in THF (2 $\mathrm{mL}$ ) was added dropwise. After $0.5 \mathrm{~h}$ at $-100{ }^{\circ} \mathrm{C}$, the reaction was allowed to warm to room temperature over $5 \mathrm{~h}$. Saturated aqueous sodium bicarbonate was added and the aqueous phase was extracted with $\mathrm{Et}_{2} \mathrm{O}(3 \times 60 \mathrm{~mL})$. The combined organic phases were washed with brine and dried over anhydrous sodium sulfate, concentrated, and purified by silica gel chromatography $\left(\mathrm{R}_{\mathrm{f}} 0.5\right.$, hexane:EtOAc $\left.=100: 5\right)$ to provide methyl $\left[15-{ }^{13} \mathrm{C}\right]-$ arachidonate $22(28 \mathrm{mg}, 53 \%)$ as a colorless oil. ${ }^{1} \mathrm{H}$ NMR $\left(500 \mathrm{MHz}, \mathrm{CDCl}_{3}\right) \delta 5.60-$ $5.545(0.5 \mathrm{H}, \mathrm{m}), 5.46-5.33(7 \mathrm{H}, \mathrm{m}), 5.29-5.24(0.5 \mathrm{H}, \mathrm{m}), 3.68(3 \mathrm{H}, \mathrm{s}), 2.87-2.81(6 \mathrm{H}, \mathrm{m})$, $2.34(2 \mathrm{H}, \mathrm{t}, \mathrm{J}=7.5 \mathrm{~Hz}), 2.14(2 \mathrm{H}, \mathrm{t}-\mathrm{d}, \mathrm{J}=14.0 \mathrm{~Hz}, \mathrm{~J}=7.0 \mathrm{~Hz}), 2.06(2 \mathrm{H}, \mathrm{t}-\mathrm{d}, \mathrm{J}=14 \mathrm{~Hz}, \mathrm{~J}=7.0$ $\mathrm{Hz}), 1.72(2 \mathrm{H}$, quin, J=7.5 Hz), 1.37-1.29 (6H, m), $0.91(3 \mathrm{H}, \mathrm{t}, \mathrm{J}=7.0 \mathrm{~Hz})$. EIMS $(\mathrm{m} / \mathrm{z})$ $319(\mathrm{M}+) .{ }^{13} \mathrm{C}$ NMR $\left(125.6 \mathrm{MHz}, \mathrm{CDCl}_{3}\right) \delta 14.3\left(\mathrm{~s}, \mathrm{CH}_{3}\right), 22.8\left(\mathrm{~s}, \mathrm{CH}_{2}\right), 25.0\left(\mathrm{~s}, \mathrm{CH}_{2}\right)$, $25.81\left(\mathrm{~s}, \mathrm{CH}_{2}\right), 25.84\left(\mathrm{~s}, \mathrm{CH}_{2}\right), 26.8\left(\mathrm{~s}, \mathrm{CH}_{2}\right), 27.4\left(\mathrm{~d}, \mathrm{~J}=42.3 \mathrm{~Hz}, \mathrm{CH}_{2}\right), 29.5$ (d, J = 1.8 $\left.\mathrm{Hz}, \mathrm{CH}_{2}\right), 31.7\left(\mathrm{~d}, \mathrm{~J}=3.7 \mathrm{~Hz}, \mathrm{CH}_{2}\right), 33.6\left(\mathrm{~s}, \mathrm{CH}_{2}\right), 51.7\left(\mathrm{~s}, \mathrm{CH}_{3}\right), 127.4,128.0,128.1$, 128.4, 128.43, 128.8, 128.82, 129.1, 129.16, 130.4, 130.7 (s, CH, Enhanced Signal), $130.9,131.0,131.3,174.3(\mathrm{~s}, \mathrm{Cq})$.

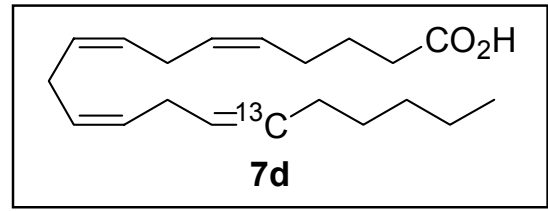

\section{$\left[15-{ }^{13} \mathrm{C}\right]$-Arachidonic acid $(7 \mathrm{~d})$}

Methyl $\left[15-{ }^{13} \mathrm{C}\right]$-arachidonate $22(10 \mathrm{mg}, 0.031 \mathrm{mmol})$ was dissolved in THF $(0.5 \mathrm{~mL})$, and cooled to $0{ }^{\circ} \mathrm{C}$. A $1 \mathrm{M}$ solution of aqueous $\mathrm{LiOH}(0.5 \mathrm{~mL}, 0.5 \mathrm{mmol})$ was added. The reaction mixture was stirred for $15 \mathrm{~h}$ at room temperature, acidified to $\mathrm{pH} 1$ with $1 \mathrm{M}$ $\mathrm{HCl}$, saturated with solid $\mathrm{NaCl}$, and extracted with $\mathrm{Et}_{2} \mathrm{O}(3 \times 60 \mathrm{~mL})$. After washing the combined organic phases with brine, drying over anhydrous sodium sulfate, and concentration under reduced pressure, purification by silica gel chromatography $\left(\mathrm{R}_{\mathrm{f}} 0.2\right.$, hexane:EtOAc $=100: 10)$ gave $\left[15-{ }^{13} \mathrm{C}\right]-\mathrm{AA} 7 \mathbf{7 d}(8.5 \mathrm{mg}, 89 \%) .{ }^{1} \mathrm{H}$ NMR $(500 \mathrm{MHz}$, $\left.\mathrm{CDCl}_{3}\right) \delta 5.60-5.545(0.5 \mathrm{H}, \mathrm{m}), 5.46-5.33(7 \mathrm{H}, \mathrm{m}), 5.29-5.24(0.5 \mathrm{H}, \mathrm{m}), 2.87-2.82(6 \mathrm{H}$, m), $2.39(2 \mathrm{H}, \mathrm{t}, \mathrm{J}=6.7 \mathrm{~Hz}), 2.16(2 \mathrm{H}, \mathrm{d}-\mathrm{t}, \mathrm{J}=14.5 \mathrm{~Hz}, \mathrm{~J}=7.0 \mathrm{~Hz}), 1.74(2 \mathrm{H}$, quin, $\mathrm{J}=7.3$ $\mathrm{Hz}), 1.41-1.25(6 \mathrm{H}, \mathrm{m}), 0.91(3 \mathrm{H}, \mathrm{t}, \mathrm{J}=7.0 \mathrm{~Hz}) ;{ }^{13} \mathrm{C}$ NMR $\left(125.6 \mathrm{MHz}, \mathrm{CDCl}_{3}\right) 130.7$ (Enhanced Signal); EIMS (m/z) $305\left(\mathrm{M}^{+}\right)$. 


\section{References:}

1) Peet, N. P.; Lentz, N. L.; Dudley, M. W.; Odgen, A. M. L.; McCarty, D. R.; Racke, M. M. J. Med Chem. 1993, 36, 4015.

2) Geneste, H.; Hesse, M. Tetrahedron 1998, 54, 15199. 